\title{
Quantum anharmonic crystal in functional integral approach
}

\author{
Yu.Kondratiev ${ }^{1,3,4}$, Yu.Kozitsky ${ }^{2}$, T.Pasurek ${ }^{1,3}$, M.Röckner ${ }^{1,3}$ \\ 1 Fakultät für Mathematik, Universität Bielefeld, D 33615 Bielefeld, Germany \\ 2 Instytut Matematyki, Uniwersytet Marii Curie-Skłodowskiej w Lublinie, \\ PL 20-031 Lublin, Poland \\ 3 Forschungszentrum BiBoS, Bielefeld, Germany \\ 4 Institute of Mathematics, Kiev, Ukraine
}

Received October 15, 2003

\begin{abstract}
A lattice model of interacting light quantum particles of mass $m$ oscillating in a crystalline field is considered in the framework of an approach based on functional integrals. The main aspects of this approach are described on an introductory level. Then a mechanism of the stabilization of this model by quantum effects is suggested. In particular, a stability condition involving $m$, the interaction intensity, and the parameters of the crystalline field is given. It is independent of the temperature and is satisfied if $m$ is small enough and/or the tunnelling frequency is big enough. It is shown that under this condition the infinite-volume correlation function decays exponentially; hence, no phase transitions can arise at all temperatures.
\end{abstract}

Key words: quantum stabilization, Gibbs states, soft mode

PACS: $05.50 .-d, 64.60 .-i$

\section{Introduction}

Structural phase transitions in ionic crystals, connected with macroscopic displacements of ionic complexes, may be triggered by the ordering of interacting localized quantum particles. A typical example here could be the ferroelectric phase transition in KDP-type compounds, in which the quantum particles are the protons oscillating along hydrogen bonds [1]. The simplest way of describing this phenomenon is to consider the subsystem of quantum particles moving in a static external field generated by heavy ions. Then the motion of the latter objects is neglected (the so-called adiabatic approximation) and each quantum particle oscillates around its equilibrium position determined by the external field. These positions are supposed to form a crystalline lattice $\mathbb{L}$. The motion of the particle with the equilibrium position at a given $\ell \in \mathbb{L}$ is described by the displacement operator $q_{\ell}$ and the momentum 
operator $p_{\ell}$, which satisfy the commutation relation

$$
\left[p_{\ell}, q_{\ell}\right]=p_{\ell} q_{\ell}-q_{\ell} p_{\ell}=1 / \mathrm{i}
$$

The quantum particles carry an electric charge, hence, their displacements create dipole moments and the inter-particle interaction potential should contain the dipole-dipole component $J_{\ell \ell^{\prime}} q_{\ell} q_{\ell^{\prime}}$. The Hamiltonian of the system of such particles which takes into account the dipole-dipole interaction only may be written in the form

$$
H=-\frac{1}{2} \sum_{\ell, \ell^{\prime}} J_{\ell \ell^{\prime}} q_{\ell} q_{\ell^{\prime}}+\sum_{\ell} H_{\ell},
$$

where the sums run through the lattice $\mathbb{L}$ and

$$
H_{\ell}=\frac{1}{2 m} p_{\ell}^{2}+\frac{a}{2} q_{\ell}^{2}+V\left(q_{\ell}\right), \quad m, a>0,
$$

is the one-particle Hamiltonian describing the particle with the equilibrium position at $q_{\ell}=0$. The third term in (1.3), in which $V: \mathbb{R} \rightarrow \mathbb{R}$, gives an anharmonic correction to the potential energy. In order for the particle to possess equilibrium at $q_{\ell}=0$ and to be localized in the vicinity of this point, the potential energy $(a / 2) \xi^{2}+V(\xi)$ ought to grow to $+\infty$ as $|\xi| \rightarrow+\infty$, to have a local extremum at $\xi=0$ and to be below bounded, i.e., to obey

$$
(a / 2) \xi^{2}+V(\xi) \geqslant v_{0}, \quad \text { for all } \xi \in \mathbb{R}
$$

with a certain real constant $v_{0}$. On the other hand, the system undergoes a structural phase transition if and only if the potential energy in (1.3) has at least two minima along with the maximum at $q_{\ell}=0$. If $V \equiv 0$, the particle is a harmonic oscillator of rigidity $a$ and reduced mass $m=m^{\text {phys }} / \hbar^{2}$, where $m^{\text {phys }}$ is the physical mass of the particle. We have included the Planck constant $\hbar$ into $m$ in order to have the commutation relation in the form of (1.1). If the function $V$ is even, the oscillations are symmetric with respect to the origin and the phase transition in the system of such particles breaks this symmetry. A typical example of $V$ is

$$
V(\xi)=b_{1} \xi^{2}+b_{2} \xi^{4}+\cdots+b_{r} \xi^{2 r}, \quad r \in \mathbb{N}, \quad r \geqslant 2 .
$$

The most known case $r=2$ has been studied for many years, see e.g., [2,3]. A detailed presentation of arguments in favor of the model (1.2) with such $V$ may be found in [4]. The model (1.1) with $V$ in the form (1.5) with $r=2$ was also used as a base of the model proposed in [5] to describe strong electron-electron correlations caused by the interaction of electrons with vibrating ions. The model (1.2) with a nonzero $V$ is called quantum anharmonic crystal.

The operators $p_{\ell}, q_{\ell}$ act in the physical Hilbert space $\mathcal{H}_{\ell}=L^{2}(\mathbb{R})$ of square integrable functions with respect to Lebesgue's measure on the real line $\mathbb{R}$. These are wave functions describing the states of the particle oscillating around the equilibrium position at $q_{\ell}=0$. Unlike the case of spin models (see e.g., [6,7]), the space $\mathcal{H}_{\ell}$ 
is infinite dimensional, which produces serious complications in the mathematical study of the model (1.1). In particular, the notion of the Gibbs state based on the so-called Kubo-Martin-Schwinger condition (see below), commonly used in the theory of quantum spin models, cannot be generalized to this case. Methods based on functional integrals have proved to be very effective in various problems of quantum theory (see e.g., [8-10]). A method of constructing Gibbs states of models like (1.1), based on functional integrals, was initiated in $[11,12]$. Now it is well elaborated, see [13-27]. The aim of this paper is to give a brief introduction into this method, accessible also to physicists. As an application, we give a detailed description of quantum effects in the model (1.1) based on the results published in [19,21-24,28].

\section{Gibbs states}

\subsection{Basic notions}

For simplicity reasons, we consider the model (1.1) on a $d$-dimensional simple cubic lattice of unit spacing, i.e., $\mathbb{L}=\mathbb{Z}^{d}$. In this case the lattice sites are $d$-dimensional integer vectors $\ell=\left(\ell_{1}, \ldots, \ell_{d}\right)$ with $\ell_{j} \in \mathbb{Z}, j=1, \ldots, d$. Regarding the interaction potential $J_{\ell \ell^{\prime}}$ we suppose that it is:

- invariant under translations on $\mathbb{L}$;

- ferroelectric, i.e., $J_{\ell \ell^{\prime}} \geqslant 0$ and $J_{\ell \ell}=0$;

- sufficiently rapidly decaying, so that $\sum_{\ell^{\prime} \in \mathbb{L}} J_{\ell \ell^{\prime}}<\infty$.

The Hamiltonian (1.2) has no direct mathematical meaning. Usually, it is "represented" by local Hamiltonians - indexed by finite subsets $\Lambda \subset \mathbb{L}$ essentially self-adjoint operators (see below) $H_{\Lambda}$ acting in the complex Hilbert space $\mathcal{H}_{\Lambda}=L^{2}\left(\mathbb{R}^{|\Lambda|}\right.$ ), where $|\Lambda|$ stands for the number of lattice sites in $\Lambda$. Elements of this space are wave functions $\psi\left(x_{\Lambda}\right)$, where $x_{\Lambda}=\left(x_{\ell}\right)_{\ell \in \Lambda}$ is the $|\Lambda|$-dimensional vector, which components $x_{\ell} \in \mathbb{R}$ are numbered by the lattice sites $\ell$ belonging to $\Lambda$. The scalar product in $\mathcal{H}_{\Lambda}$ is defined as follows:

$$
\langle\psi \mid \phi\rangle_{\mathcal{H}_{\Lambda}}=\overline{\langle\phi \mid \psi\rangle_{\mathcal{H}_{\Lambda}}}=\int_{\mathbb{R}^{|\Lambda|}} \overline{\psi\left(x_{\Lambda}\right)} \phi\left(x_{\Lambda}\right) \prod_{\ell \in \Lambda} \mathrm{d} x_{\ell},
$$

where $\bar{z}$ means complex conjugation. Suppose that a linear operator $Q: \mathcal{H}_{\Lambda} \rightarrow \mathcal{H}_{\Lambda}$ acts on a given $\phi \in \mathcal{H}_{\Lambda}$. We write the scalar product of $Q \phi$ and a given $\psi \in \mathcal{H}_{\Lambda}$ in the form

$$
\langle\psi \mid Q \phi\rangle_{\mathcal{H}_{\Lambda}}=\langle\psi|Q| \phi\rangle_{\mathcal{H}_{\Lambda}}, \quad\langle Q \psi \mid \phi\rangle_{\mathcal{H}_{\Lambda}}=\overline{\langle\phi|Q| \psi\rangle_{\mathcal{H}_{\Lambda}}}
$$

In infinite dimensional spaces linear operators need not be defined on the whole space. Given a linear operator $Q$, by $\mathcal{D}(Q)$ we denote its domain, i.e., the set of functions $\psi \in \mathcal{H}_{\Lambda}$ on which the action of $Q$ is defined. A linear operator $Q: \mathcal{H}_{\Lambda} \rightarrow$ $\mathcal{H}_{\Lambda}$ is called bounded if there exists a constant $C>0$ such that

$$
\langle\psi|Q| \psi\rangle_{\mathcal{H}_{\Lambda}} \leqslant C\langle\psi \mid \psi\rangle_{\mathcal{H}_{\Lambda}},
$$


for all $\psi \in \mathcal{D}(Q)$. The least such $C$ characterizes the operator - it is its norm $\|Q\|$. We suppose that, for every bounded operator, $\mathcal{D}(Q)=\mathcal{H}_{\Lambda}$. The operators which fail to obey (2.1) are called unbounded. Each unbounded operator considered in this article is densely defined, i.e., the closure of its domain coincides with $\mathcal{H}_{\Lambda}$. For a bounded operator $Q$, its adjoint operator $Q^{*}: \mathcal{H}_{\Lambda} \rightarrow \mathcal{H}_{\Lambda}$ is defined by the relation

$$
\langle\psi|Q| \phi\rangle_{\mathcal{H}_{\Lambda}}=\overline{\left\langle\phi\left|Q^{*}\right| \psi\right\rangle_{\mathcal{H}_{\Lambda}}},
$$

which has to hold for all $\psi, \phi \in \mathcal{H}_{\Lambda}$. It is also a bounded operator and $\|Q\|=\left\|Q^{*}\right\|$. A bounded operator $Q$ is called self-adjoint if $Q^{*}=Q$. For unbounded operators, the definition of self-adjointness is a bit more complicated. Given $Q, Q^{\prime}$, suppose $\mathcal{D}(Q) \subset \mathcal{D}\left(Q^{\prime}\right)$ and, for every $\psi \in \mathcal{D}(Q)$, one has $Q \psi=Q^{\prime} \psi$. Then the operator $Q^{\prime}$ is called an extension of $Q$ to the set $\mathcal{D}\left(Q^{\prime}\right)$. Given a densely defined operator $Q$, the operator $S: \mathcal{H}_{\Lambda} \rightarrow \mathcal{H}_{\Lambda}$ with the maximal domain obeying the condition

$$
\langle\psi|Q| \phi\rangle_{\mathcal{H}_{\Lambda}}=\overline{\langle\phi|S| \psi\rangle_{\mathcal{H}_{\Lambda}}}, \quad \text { for all } \phi \in \mathcal{D}(Q) \text { and } \psi \in \mathcal{D}(S),
$$

is called adjoined to $Q$, i.e., $S=Q^{*}$. The operator $Q$ is called symmetric if $Q^{*}$ is its extension; it is called self-adjoint if $Q=Q^{*}$. Finally, such $Q$ is called essentially self-adjoined if it possesses a unique self-adjoint extension.

By $\mathfrak{C}_{\Lambda}$ we denote the set of all bounded operators $Q: \mathcal{H}_{\Lambda} \rightarrow \mathcal{H}_{\Lambda}$. This set is equipped with the above introduced norm $\|\cdot\|$, with the addition and multiplication by complex numbers and with the multiplication $Q Q^{\prime}$ such that

$$
\left\|Q Q^{\prime}\right\| \leqslant\|Q\|\left\|Q^{\prime}\right\|, \quad\|Q\|=\left\|Q^{*}\right\|
$$

Then $\mathfrak{C}_{\Lambda}$ is a $C^{*}$-algebra (see [29] for more details). Its unity is the identity operator $I$ which maps each $\psi \in \mathcal{H}_{\Lambda}$ into itself. A self-adjoint operator $Q \in \mathfrak{C}_{\Lambda}$ is called positive if,

$$
\langle\psi|Q| \psi\rangle_{\mathcal{H}_{\Lambda}} \geqslant 0, \quad \text { for every } \psi \in \mathcal{H}_{\Lambda} .
$$

Let $\omega: \mathfrak{C}_{\Lambda} \rightarrow \mathbb{C}$ be a bounded linear functional on the algebra $\mathfrak{C}_{\Lambda}$, i.e., such that there exists a constant $\omega_{0} \geqslant 0$ for which and for any $Q \in \mathfrak{C}_{\Lambda}$,

$$
|\omega(Q)| \leqslant \omega_{0}\|Q\|
$$

A linear functional $\omega: \mathfrak{C}_{\Lambda} \rightarrow \mathbb{C}$ is called a state on $\mathfrak{C}_{\Lambda}$ if: (a) it is normalized, i.e., $\omega(I)=1$; (b) it is positive, i.e., $\omega(Q) \geqslant 0$ for any positive $Q \in \mathfrak{C}_{\Lambda}$.

Each base of $\mathcal{H}_{\Lambda}$ - a complete orthonormal system of wave functions - should be an infinite countable set. Let $\left\{\psi_{n}\right\}_{n \in \mathbb{N}}$ be such a base. For a linear operator $T: \mathcal{H}_{\Lambda} \rightarrow \mathcal{H}_{\Lambda}$, suppose that $\left\{\psi_{n}\right\}_{n \in \mathbb{N}}$ is contained in its domain. Set

$$
\operatorname{trace}(T)=\sum_{n=1}^{\infty}\left\langle\psi_{n}|T| \psi_{n}\right\rangle_{\mathcal{H}_{\Lambda}} .
$$

The above series need not be convergent. If it is absolutely convergent, the operator $T$ is bounded, hence $\mathcal{D}(T)=\mathcal{H}_{\Lambda}$, and (2.2) has the same value for any orthonormal 
base of $\mathcal{H}_{\Lambda}$. In this case we say that $T$ is of trace class and the number trace $(T)$ is called its trace. In the case of spin models the Hilbert spaces are finite dimensional and all linear operators are of trace class. For any bounded operator $Q$ and any trace class operator $T$, the operators $Q T$ and $T Q$ are of trace class and

$$
|\operatorname{trace}(Q T)| \leqslant\|Q\||\operatorname{trace}(T)|, \quad|\operatorname{trace}(T Q)| \leqslant\|Q\||\operatorname{trace}(T)| \text {. }
$$

The latter estimate follows from the former one and from the cyclicity

$$
\operatorname{trace}[A B C]=\operatorname{trace}[B C A]=\operatorname{trace}[C A B] .
$$

For a finite subset $\Lambda \subset \mathbb{L}$, the local Hamiltonian corresponding to the zero boundary conditions outside of $\Lambda$ is set as follows:

$$
H_{\Lambda}(0)=-\frac{1}{2} \sum_{\ell, \ell^{\prime} \in \Lambda} J_{\ell \ell^{\prime}} q_{\ell} q_{\ell^{\prime}}+\sum_{\ell \in \Lambda} H_{\ell}
$$

where $J_{\ell \ell^{\prime}}$ and $H_{\ell}$ are the same as in (1.2), (1.3). A standard fact from the theory of such operators (see e.g., [30]) is that, for any $\beta>0$, the operator $\exp \left(-\beta H_{\Lambda}(0)\right)$ is of trace class. At the same time, the operators $q_{\ell}, p_{\ell}$, as well as the Hamiltonians $H_{\ell}, H_{\Lambda}(0)$, defined in the Hilbert space $\mathcal{H}_{\Lambda}$, are unbounded. Usually, such operators are defined on the sets of differentiable functions with a sufficiently fast falloff (Schwartz spaces of test functions, see e.g., [31]). Then one proves that they are essentially self-adjoint.

In a standard situation, it is enough to consider local Hamiltonians indexed by boxes. Given $L \in \mathbb{N}$, the box $\Lambda_{L}$ is

$$
\Lambda_{L}=(-L, L]^{d} \bigcap \mathbb{L}
$$

We shall often drop the subscript $L$ assuming that a given $\Lambda$ is of the form (2.6) with some $L \in \mathbb{N}$. The assumed properties of $J_{\ell \ell^{\prime}}$ imply that there exists a function $\Phi: \mathbb{R}_{+} \rightarrow \mathbb{R}_{+} \stackrel{\text { def }}{=}[0,+\infty)$ such that

$$
J_{\ell \ell^{\prime}}=\Phi\left(\left|\ell-\ell^{\prime}\right|\right)
$$

where $\left|\ell-\ell^{\prime}\right|=\sqrt{\left|\ell_{1}-\ell_{1}^{\prime}\right|^{2}+\cdots+\left|\ell_{d}-\ell_{d}^{\prime}\right|^{2}}$ is the Euclidean distance on the lattice $\mathbb{L}$. Given $\Lambda_{L}$, we set

$$
\left|\ell-\ell^{\prime}\right|_{\Lambda_{L}}=\sqrt{\left|\ell_{1}-\ell_{1}^{\prime}\right|_{L}^{2}+\cdots+\left|\ell_{d}-\ell_{d}^{\prime}\right|_{L}^{2}}, \quad \ell, \ell^{\prime} \in \Lambda_{L}
$$

where $\left|\ell_{j}-\ell_{j}^{\prime}\right|_{L} \stackrel{\text { def }}{=} \min \left\{\left|\ell_{j}-\ell_{j}^{\prime}\right|, 2 L-\left|\ell_{j}-\ell_{j}^{\prime}\right|\right\}$ is the distance on the torus which one obtains by identifying the opposite walls of the box $\Lambda_{L}$. It is invariant under translations on this torus. For a box $\Lambda(2.6)$ and $\ell, \ell^{\prime} \in \Lambda$, we set

$$
J_{\ell \ell^{\prime}}^{\Lambda}=\Phi\left(\left|\ell-\ell^{\prime}\right|_{\Lambda}\right)
$$


where $\Phi$ is the same as in (2.7). Then the potential $J_{\ell \ell^{\prime}}^{\Lambda}$ is invariant under translations on the torus. The local Hamiltonian corresponding to the periodic conditions on the boundaries of $\Lambda$ is

$$
H_{\Lambda}(p)=-\frac{1}{2} \sum_{\ell, \ell^{\prime} \in \Lambda} J_{\ell \ell^{\prime}}^{\Lambda} q_{\ell} q_{\ell^{\prime}}+\sum_{\ell \in \Lambda} H_{\ell} .
$$

As above, the Hamiltonian $H_{\Lambda}(p)$ is defined on the set of test functions, it is essentially self-adjoint. The operator $\exp \left(-\beta H_{\Lambda}(p)\right)$ is of trace class for any $\beta>0$, which allows us to set

$$
Z_{\beta, \Lambda}(0)=\operatorname{trace}\left[\exp \left(-\beta H_{\Lambda}(0)\right)\right], \quad Z_{\beta, \Lambda}(p)=\operatorname{trace}\left[\exp \left(-\beta H_{\Lambda}(p)\right)\right] .
$$

These are the local partition functions corresponding to the zero and periodic boundary conditions respectively. Furthermore, we set

$$
R_{\beta, \Lambda}(0)=\exp \left(-\beta H_{\Lambda}(0)\right) / Z_{\beta, \Lambda}(0), \quad R_{\beta, \Lambda}(p)=\exp \left(-\beta H_{\Lambda}(p)\right) / Z_{\beta, \Lambda}(p) .
$$

Both these operators are of trace class and

$$
\operatorname{trace}\left(R_{\beta, \Lambda}(0)\right)=\operatorname{trace}\left(R_{\beta, \Lambda}(p)\right)=1 .
$$

They are density matrices corresponding to the zero and periodic boundary conditions respectively. The local Gibbs states of our model, corresponding to the zero and periodic boundary conditions, appear to be the following linear functionals on the algebra $\mathfrak{C}_{\Lambda}$

$$
\varrho_{\beta, \Lambda}^{(0)}(Q)=\operatorname{trace}\left[Q R_{\beta, \Lambda}(0)\right], \quad \varrho_{\beta, \Lambda}^{(p)}(Q)=\operatorname{trace}\left[Q R_{\beta, \Lambda}(p)\right], \quad Q \in \mathfrak{C}_{\Lambda} .
$$

By (2.13), they are normalized; by (2.3) and (2.13), they are bounded with $\omega_{0}=1$ in both cases. The elements of $\mathfrak{C}_{\Lambda}$ are called local observables and $\mathfrak{C}_{\Lambda}$ itself is called the algebra of local observables. Here local means related with a finite subset of the lattice $\mathbb{L}$. Thermodynamic quantities are obtained in the limit $\Lambda \rightarrow \mathbb{L}$, which can be reached by letting $L \rightarrow+\infty$ (see (2.6)).

In the Heisenberg approach to quantum mechanics, the dynamics of the system described by the local Hamiltonians (2.5), (2.10) is generated by the following time maps acting on the algebra of observables $\mathfrak{C}_{\Lambda}$

$$
\mathfrak{a}_{0, \Lambda}^{t}(Q)=U_{0, \Lambda}^{t} Q\left[U_{0, \Lambda}^{t}\right]^{-1}, \quad \mathfrak{a}_{p, \Lambda}^{t}(Q)=U_{p, \Lambda}^{t} Q\left[U_{p, \Lambda}^{t}\right]^{-1}, \quad Q \in \mathfrak{C}_{\Lambda},
$$

where $t \in \mathbb{R}$ is time and

$$
U_{0, \Lambda}^{t}=\exp \left(\mathrm{it} H_{\Lambda}(0)\right), \quad U_{p, \Lambda}^{t}=\exp \left(\mathrm{i} t H_{\Lambda}(p)\right) .
$$

Sometimes, (2.15) are called Heisenberg representations of $Q$. Since the Hamiltonians $H_{\Lambda}(0), H_{\Lambda}(p)$ are essentially self-adjoint, all $U_{0, \Lambda}^{t}, U_{p, \Lambda}^{t}, t \in \mathbb{R}$ are unitary operators on the Hilbert space $\mathcal{H}_{\Lambda}$. Therefore, the time maps (2.15) are norm-preserving

$$
\left\|\mathfrak{a}_{0, \Lambda}^{t}(Q)\right\|=\|Q\|, \quad\left\|\mathfrak{a}_{p, \Lambda}^{t}(Q)\right\|=\|Q\|, \quad Q \in \mathfrak{C}_{\Lambda},
$$


and for any $t, s \in \mathbb{R}$,

$$
\mathfrak{a}_{0, \Lambda}^{t}\left(\mathfrak{a}_{0, \Lambda}^{s}(Q)\right)=\mathfrak{a}_{0, \Lambda}^{t+s}(Q), \quad \mathfrak{a}_{p, \Lambda}^{t}\left(\mathfrak{a}_{p, \Lambda}^{s}(Q)\right)=\mathfrak{a}_{p, \Lambda}^{t+s}(Q), \quad Q \in \mathfrak{C}_{\Lambda} .
$$

By (2.15), (2.16), $\mathfrak{a}_{0, \Lambda}^{0}(Q)=\mathfrak{a}_{p, \Lambda}^{0}(Q)=Q$, which means that such maps constitute one-parameter groups. These groups generate unitary time evolutions of the corresponding algebras of observables. Given $Q_{1}, \ldots, Q_{n} \in \mathfrak{C}_{\Lambda}, n \in \mathbb{N}$, we set

$$
\begin{array}{ll}
G_{Q_{1}, \ldots, Q_{n}}^{0, \beta, \Lambda}\left(t_{1}, \ldots, t_{n}\right)=\varrho_{\beta, \Lambda}^{(0)}\left\{\mathfrak{a}_{0, \Lambda}^{t_{1}}\left(Q_{1}\right) \cdots \mathfrak{a}_{0, \Lambda}^{t_{n}}\left(Q_{n}\right)\right\}, & t_{1}, \ldots, t_{n} \in \mathbb{R} \\
G_{Q_{1}, \ldots, Q_{n}}^{p, \beta,}\left(t_{1}, \ldots, t_{n}\right)=\varrho_{\beta, \Lambda}^{(p)}\left\{\mathfrak{a}_{p, \Lambda}^{t_{1}}\left(Q_{1}\right) \cdots \mathfrak{a}_{p, \Lambda}^{t_{n}}\left(Q_{n}\right)\right\}, & t_{1}, \ldots, t_{n} \in \mathbb{R} .
\end{array}
$$

One can consider the left-hand sides of (2.18), (2.19) as functions of real variables $t_{1}, \ldots, t_{n}$. They are called Green functions corresponding to the operators (observables) $Q_{1}, \ldots, Q_{n}$ and to the zero and periodic boundary conditions, respectively. The analytic properties of these functions play a crucial role in our theory. They are described by the following statement which was proved in [22], see Lemma 2.1. For a complex number $z=x+$ i $y$, we write $x=\Re(z), y=\Im(z)$. Set

$$
\mathcal{O}_{n}^{\beta}=\left\{\left(t_{1}, \ldots, t_{n}\right) \in \mathbb{C}^{n} \mid 0<\Im\left(t_{1}\right)<\cdots<\Im\left(t_{n}\right)<\beta\right\} .
$$

Proposition 1 For every $Q_{1}, \ldots, Q_{n} \in \mathfrak{C}_{\Lambda}$,

(a) $G_{Q_{1}, \ldots, Q_{n}}^{0, \beta, \Lambda}$ can be extended to a function analytic on $\mathcal{O}_{n}^{\beta}$;

(b) this extension (which will also be denoted by $G_{Q_{1}, \ldots, Q_{n}}^{0, \beta, \Lambda}$ ) is continuous on the closure $\overline{\mathcal{O}}_{n}^{\beta}$ of $\mathcal{O}_{n}^{\beta}$; moreover, for all $\left(t_{1}, \ldots, t_{n}\right) \in \overline{\mathcal{O}}_{n}^{\beta}$,

$$
\left|G_{Q_{1}, \ldots, Q_{n}}^{0, \beta, \Lambda}\left(t_{1}, \ldots, t_{n}\right)\right| \leqslant\left\|Q_{1}\right\| \cdots\left\|Q_{n}\right\|
$$

(c) for every $\xi_{1}, \ldots, \xi_{n} \in \mathbb{R}$, the set

$$
\mathcal{O}_{n}^{\beta}\left(\xi_{1}, \ldots, \xi_{n}\right) \stackrel{\text { def }}{=}\left\{\left(t_{1}, \ldots, t_{n}\right) \in \mathcal{O}_{n}^{\beta} \mid \Re\left(t_{j}\right)=\xi_{j}, \quad j=1, \ldots, n\right\},
$$

is such that, for any two functions $F, G$ analytic on $\mathcal{O}_{n}^{\beta}$, the equality $F=G$ for all $\left(t_{1}, \ldots, t_{n}\right) \in \mathcal{O}_{n}^{\beta}\left(\xi_{1}, \ldots, \xi_{n}\right)$ implies that these functions are equal on the whole $\mathcal{O}_{n}^{\beta}$.

The functions (2.19) possess the same properties.

The property described by claim (c) may be also interpreted as follows: every function, analytic on $\mathcal{O}_{n}^{\beta}$, can be uniquely determined by the values which it takes at fixed real parts of its arguments. Let us set them to be zero and consider

$$
\begin{aligned}
& \Gamma_{Q_{1}, \ldots, Q_{n}}^{0, \beta, \Lambda}\left(\tau_{1}, \ldots, \tau_{n}\right)=G_{Q_{1}, \ldots, Q_{n}}^{0, \beta, \Lambda}\left(\mathrm{i} \tau_{1}, \ldots, \mathrm{i} \tau_{n}\right), \\
& \Gamma_{Q_{1}, \ldots, Q_{n}}^{p,,, Q_{1}}\left(\tau_{1}, \ldots, \tau_{n}\right)=G_{Q_{1}, \ldots, Q_{n}}^{p, \beta,}\left(\mathrm{i} \tau_{1}, \ldots, \mathrm{i} \tau_{n}\right),
\end{aligned}
$$

where we suppose that (c.f., (2.20))

$$
0 \leqslant \tau_{1} \leqslant \cdots \leqslant \tau_{n} \leqslant \beta
$$


The left-hand sides of (2.23), (2.24) are called Matsubara functions corresponding to the observables $Q_{1}, \ldots, Q_{n}$ and to the zero and periodic boundary conditions respectively. By claim (b), the Matsubara functions are continuous on their domains; by claim (c), they completely determine the corresponding Green functions (2.18), (2.19). In what follows, the Green functions possess analytic continuations to complex values of time and their values at imaginary time determine uniquely the values at real time. The latter fact also takes place in quantum field theory where the points of the Minkowski space corresponding to imaginary time may be interpreted as points of a Euclidean space. The approach which exploits this fact is known as Euclidean quantum field theory.

The Matsubara functions possess one more property implied by Proposition 1 . Given $\tau, \tau^{\prime} \in[0, \beta]$, we set

$$
\tau \stackrel{\circ}{+} \tau^{\prime}= \begin{cases}\tau+\tau^{\prime}, & \tau+\tau^{\prime} \leqslant \beta, \\ \tau+\tau^{\prime}-\beta, & \tau+\tau^{\prime}>\beta .\end{cases}
$$

This operation is called addition of modulo $\beta$. It may be interpreted as addition on the circle of length $\beta$ obtained by identifying the endpoints of $[0, \beta]$. For $t=\theta+\mathrm{i} \tau$, $\theta \in \mathbb{R}, \tau \in[0, \beta]$ and some $\tau^{\prime} \in[0, \beta]$, we write $t \stackrel{\circ}{+} \mathrm{i} \tau^{\prime}$ meaning $\theta+\mathrm{i}\left(\tau \stackrel{\circ}{+} \tau^{\prime}\right)$.

Proposition 2 The Green functions possess the following property

$$
\begin{aligned}
& G_{Q_{1}, \ldots, Q_{n}}^{0, \beta, \Lambda}\left(t_{1}, \ldots, t_{n}\right)=G_{Q_{1}, \ldots, Q_{n}}^{0, \beta, \Lambda}\left(t_{1} \stackrel{\circ}{+} \mathrm{i} \beta, \ldots, t_{n} \stackrel{\circ}{+} \mathrm{i} \beta\right), \\
& G_{Q_{1}, \ldots, Q_{n}}^{p, \beta, \Lambda}\left(t_{1}, \ldots, t_{n}\right)=G_{Q_{1}, \ldots, Q_{n}}^{p, \beta, \Lambda}\left(t_{1} \stackrel{\circ}{+} \mathrm{i} \beta, \ldots, t_{n} \stackrel{\circ}{+} \mathrm{i} \beta\right) .
\end{aligned}
$$

In particular,

$$
\begin{aligned}
& \Gamma_{Q_{1}, \ldots, Q_{n}}^{0, \beta, \Lambda}\left(\tau_{1}, \ldots, \tau_{n}\right)=\Gamma_{Q_{1}, \ldots, Q_{n}}^{0, \beta, \Lambda}\left(\tau_{1} \stackrel{\circ}{+} \beta, \ldots, \tau_{n} \stackrel{\circ}{+} \beta\right), \\
& \Gamma_{Q_{1}, \ldots, Q_{n}}^{p, \beta, \Lambda}\left(\tau_{1}, \ldots, \tau_{n}\right)=\Gamma_{Q_{1}, \ldots, Q_{n}}^{p, \beta, \Lambda}\left(\tau_{1} \stackrel{\circ}{+} \beta, \ldots, \tau_{n} \stackrel{\circ}{+} \beta\right) .
\end{aligned}
$$

\subsection{KMS condition and Høegh-Krohn theorem}

Let us consider the property (2.27) in the case of $n=2$ and the zero boundary conditions. For any $A, B \in \mathfrak{C}_{\Lambda}$ and any $t \in \mathbb{R}$, one has

$$
\begin{aligned}
G_{A, B}^{0, \beta, \Lambda}(0, t \stackrel{\circ}{+} \mathrm{i} \beta)= & \operatorname{trace}\left\{A \exp \left(\mathrm{it} H_{\Lambda}(0)-\beta H_{\Lambda}(0)\right) B\right. \\
& \left.\times \exp \left(-\mathrm{i} t H_{\Lambda}(0)+\beta H_{\Lambda}(0)\right) \exp \left(-\beta H_{\Lambda}(0)\right)\right\} / Z_{\beta, \Lambda}(0) \\
= & \operatorname{trace}\left\{A \exp \left(-\beta H_{\Lambda}(0)\right) \exp \left(\mathrm{i} t H_{\Lambda}(0)\right) B \exp \left(-\mathrm{i} t H_{\Lambda}(0)\right\} / Z_{\beta, \Lambda}(0)\right. \\
= & \operatorname{trace}\left\{\exp \left(\mathrm{i} t H_{\Lambda}(0)\right) B \exp \left(-\mathrm{i} t H_{\Lambda}(0) A \exp \left(-\beta H_{\Lambda}(0)\right)\right\} / Z_{\beta, \Lambda}(0)\right. \\
= & \varrho_{\beta, \Lambda}^{(0)}\left(\mathfrak{a}_{0, \Lambda}^{t}(B) A\right)=\varrho_{\beta, \Lambda}^{(0)}\left(\mathfrak{a}_{0, \Lambda}^{t}(B) \mathfrak{a}_{0, \Lambda}^{0}(A)\right) \\
= & G_{B, A}^{0, \beta, \Lambda}(t, 0) .
\end{aligned}
$$

Here we have used (2.4) and (2.14)-(2.17). The above computations may be repeated for the Green functions corresponding to the periodic boundary conditions. In what follows, Proposition 1 and the above computations yield the next statement. 
Proposition 3 The local Gibbs states (2.14) possess the following property. For any $A, B \in \mathfrak{C}_{\Lambda}$, there exist functions $F_{A, B}^{(0)}(z), F_{A, B}^{(p)}(z)$, analytic in the open strip $\{t+\mathrm{i} \tau \mid t \in \mathbb{R}, \tau \in(0, \beta)\}$ and continuous on its closure, which satisfy the conditions

$$
\begin{array}{ll}
F_{A, B}^{(0)}(t)=\varrho_{\beta, \Lambda}^{(0)}\left(A \mathfrak{a}_{0, \Lambda}^{t}(B)\right), & F_{A, B}^{(0)}(t+\stackrel{\circ}{\mathrm{i} \beta})=\varrho_{\beta, \Lambda}^{(0)}\left(\mathfrak{a}_{0, \Lambda}^{t}(B) A\right), \\
F_{A, B}^{(p)}(t)=\varrho_{\beta, \Lambda}^{(p)}\left(A \mathfrak{a}_{p, \Lambda}^{t}(B)\right), & F_{A, B}^{(p)}(t \stackrel{\circ}{+} \mathrm{i} \beta)=\varrho_{\beta, \Lambda}^{(p)}\left(\mathfrak{a}_{p, \Lambda}^{t}(B) A\right) .
\end{array}
$$

Proof: Take $F_{A, B}^{(0)}(z)=G_{A, B}^{0, \beta, \Lambda}(0, z)$ and $F_{A, B}^{(p)}(z)=G_{A, B}^{p, \beta, \Lambda}(0, z)$.

The property described by Proposition 3 reflects a consistency between the dynamics and the states $\varrho_{\beta, \Lambda}^{(0)}, \varrho_{\beta, \Lambda}^{(p)}$. It comes from the fact that the dynamics, defined by (2.15), (2.16), and the density matrices, defined by (2.12)-(2.14), are determined by one and the same Hamiltonian. Such a consistency, natural for local Gibbs states, cannot be transferred automatically to the infinite-volume case. First of all due to the fact that in this case one has no Hamiltonians (as operators) to determine the dynamics and the states. Moreover, to describe such phenomena as phase transitions one has to allow multiplicity of infinite-volume Gibbs states corresponding to one and the same heuristic Hamiltonian (1.1). If the infinite-volume dynamics can be constructed as the limit of the time maps (2.15), (2.16), it would be quite natural to expect that the limiting Gibbs states and time maps are consistent in the sense of Proposition 3. Therefore, one can define an infinite-volume Gibbs state as the state satisfying a condition like (2.29). The latter is called the Kubo-MartinSchwinger condition, see [29] for detailed explanations, and [32] for the latest development. Let us give a definition of a Kubo-Martin-Schwinger state. For $\Lambda, \Lambda^{\prime}$ such that $\Lambda \subset \Lambda^{\prime}$, one may consider the elements of $\mathfrak{C}_{\Lambda}$ as elements of $\mathfrak{C}_{\Lambda^{\prime}}$. Indeed, for $Q \in \mathfrak{C}_{\Lambda}$, we set $Q^{\prime}=Q \otimes I$, where $I \in \mathfrak{C}_{\Lambda^{\prime} \backslash \Lambda}$ is the identity operator. Then, for $\psi^{\prime}\left(x_{\Lambda^{\prime}}\right)=\psi\left(x_{\Lambda}\right) \cdot \phi\left(x_{\Lambda^{\prime} \backslash \Lambda}\right)$,

$$
Q^{\prime} \psi^{\prime}\left(x_{\Lambda^{\prime}}\right)=\left(Q \psi\left(x_{\Lambda}\right)\right) \cdot \phi\left(x_{\Lambda^{\prime} \backslash \Lambda}\right) .
$$

One can ignore the difference between the above $Q^{\prime}$ and $Q$ and consider $Q$ as an element of the "bigger" algebra $\mathfrak{C}_{\Lambda^{\prime}}$, which acts on "its own" variables. This allows us to consider the "smaller" algebra $\mathfrak{C}_{\Lambda}$ as a subalgebra of the "bigger" one. Now, for a sequence of boxes $\mathcal{L}$, which exhausts the lattice $\mathbb{L}$, we set

$$
\mathfrak{C}_{\mathrm{loc}}=\bigcup_{\Lambda \in \mathcal{L}} \mathfrak{C}_{\Lambda}
$$

which is called the local algebra of observables. This algebra contains all bounded operators on the Hilbert spaces $\mathcal{H}_{\Lambda}, \Lambda \in \mathcal{L}$. It need not be complete - some fundamental sequences in $\mathfrak{C}_{\text {loc }}$ may have no limits in this algebra. The completion $\mathfrak{C}$ of the algebra $\mathfrak{C}_{\text {loc }}$ is called the quasi-local algebra of observables. It is a Banach algebra and contains the latter one as a dense subset. The dynamics of the model is defined by a group $\mathfrak{A}=\left\{\mathfrak{a}^{t}\right\}_{t \in \mathbb{R}}$ of time maps, which have to be obtained as limits of the maps (2.15), if those ones do exist. We recall that a state on $\mathfrak{C}$ is a positive normalized functional on this algebra. Since each state is norm-continuous, it may be uniquely determined by its values on a norm-dense subset of $\mathfrak{C}$, i.e., on the local algebra $\mathfrak{C}_{\text {loc }}$. 
Definition 4 A state $\omega$ on the quasi-local algebra $\mathfrak{C}$ is called a $\beta$-KMS state corresponding to the group of time maps $\mathfrak{A}$, if for any $A, B \in \mathfrak{C}$, there exists a function $F_{A, B}(z)$, analytic in the open strip $\{t+\mathrm{i} \tau \mid t \in \mathbb{R}, \quad \tau \in(0, \beta)\}$ and continuous on its closure, which satisfies the $\beta-K M S$ condition

$$
F_{A, B}(t)=\omega\left(A \mathfrak{a}^{t}(B)\right), \quad F_{A, B}(t \stackrel{\circ}{+} \beta)=\omega\left(\mathfrak{a}^{t}(B) A\right) .
$$

Such $\beta$-KMS states are consistent with the dynamics defined by $\mathfrak{A}$. If a state is unique for a given $\beta$, then there exists exactly one phase (the state itself) at this temperature. For an observable $A \in \mathfrak{C}$, its value in this phase is just $\omega(A)$. If, for a given $\beta$, one has more than one $\beta$-KMS state, then a phase transition occurs. Let $\omega$ and $\omega^{\prime}$ be two different $\beta$-KMS states. Then their convex combination

$$
\omega^{\prime \prime}=\theta \omega+(1-\theta) \omega^{\prime}, \quad \theta \in[0,1]
$$

is also a $\beta$-KMS state. Such a state is called a mixture of the states $\omega$ and $\omega^{\prime}$ if $\theta \in(0,1)$. A $\beta$-KMS state is called a pure $\beta$-KMS state if it is not a mixture of any other $\beta$-KMS states. Only pure $\beta$-KMS states have physical meaning, they are called phases. In this setting the phase transition corresponds to non-uniqueness of pure $\beta$-KMS states. The above scheme does work in the case of spin models, where one can construct, by taking the limit of the maps (2.15), the group of time maps $\mathfrak{A}$. The situation in the case of the model (1.2) is different - there are no infinite-volume dynamics, hence, there are no $\beta$-KMS states, at least in the sense of Definition 4. This difficulty is a principle one, it demands new concepts in defining the notion of equilibrium state. The approach we describe in this publication presents such a concept; it may be outlined as follows. Like in classical statistical physics, one defines local Gibbs states as probability measures, but now on infinite dimensional spaces. Then one defines the phases by means of the so-called equilibrium equation, also known as the Dobrushin-Lanford-Ruelle (DLR) equation - the central notion of classical statistical physics [33]. This approach is based on the Høegh-Krohn theorem which establishes the one-to-one correspondence between the local Gibbs states (2.14) and the mentioned measures. A crucial role here is played by the Matsubara functions corresponding to multiplication operators and by Proposition 1. Given $\Lambda$ and a bounded function $F: \mathbb{R}^{|\Lambda|} \rightarrow \mathbb{R}$, we define the operator

$$
(F \psi)\left(x_{\Lambda}\right)=F\left(x_{\Lambda}\right) \psi\left(x_{\Lambda}\right),
$$

which action on $\psi \in \mathcal{H}_{\Lambda}$ is multiplication by the function $F$. This operator is bounded since the function $F$ is so. The displacement operator $q_{\ell}$ is a multiplication operator but it is unbounded. Given $\Lambda$, by $\mathfrak{M}_{\Lambda}$ we denote the set of all multiplication operators by bounded continuous functions $F: \mathbb{R}^{|\Lambda|} \rightarrow \mathbb{R}$. For such a function, we set

$$
\|F\|_{\infty}=\sup _{x_{\Lambda} \in \mathbb{R}^{|\Lambda|}}\left|F\left(x_{\Lambda}\right)\right|
$$

Then for every $F \in \mathfrak{M}_{\Lambda}$, the norm $\|F\|$ of this operator is equal to the above supremum norm of the corresponding function. 
For our purposes, there is no need in employing the Høegh-Krohn theorem in its entirety, so we formulate only a simplified version. Take $n \in \mathbb{N}$ and $F_{1}, \ldots, F_{n} \in \mathfrak{M}_{\Lambda}$.

Proposition 5 The set of operators

$$
\mathfrak{a}_{0, \Lambda}^{t_{1}}\left(F_{1}\right) \cdots \mathfrak{a}_{0, \Lambda}^{t_{n}}\left(F_{n}\right), \quad F_{1}, \ldots, F_{n} \in \mathfrak{M}_{\Lambda}, \quad t_{1}, \ldots, t_{n} \in \mathbb{R},
$$

with all possible choices of $n$, the operators and time moments, is a state determining set. This means if two states on $\mathfrak{C}_{\Lambda}$ have the same values on all such operators, then they coincide. The same property have the operators (2.34) corresponding to the periodic boundary conditions.

The essence of the above statement is that a state on $\mathfrak{C}_{\Lambda}$ is completely determined by its values on the operators (2.34). But, the values of the local Gibbs states (2.14) on such operators are the Green functions (2.18), (2.19) corresponding to multiplication operators. Thus, the states (2.14) are completely determined by the Green functions corresponding to multiplication operators by bounded continuous functions. Finally, in view of Proposition 1, such Green functions are completely determined by the Matsubara functions (2.23), (2.24) corresponding to the mentioned multiplication operators. In what follows, we have the following corollary of the Høegh-Krohn theorem.

Corollary 6 The Matsubara functions (2.23), (2.24) corresponding to all multiplication operators by bounded continuous functions completely determine the local Gibbs states (2.14).

In the functional integral approach the Matsubara functions corresponding to multiplication operators are written as moments of certain probability measures, which we describe in the next subsection.

\subsection{Euclidean Gibbs measures}

Given $\beta>0$, by $\mathcal{E}$ we denote the real Hilbert space $L^{2}[0, \beta]$ of all real valued functions which are square integrable on $[0, \beta]$. For $x, y \in \mathcal{E}$, the scalar product and the norm are

$$
(x, y)_{\mathcal{E}}=\int_{0}^{\beta} x(\tau) y(\tau) \mathrm{d} \tau, \quad\|x\|_{\mathcal{E}}=\sqrt{(x, x)_{\mathcal{E}}}
$$

The following set of functions

$$
e_{k}(\tau)= \begin{cases}\sqrt{2 / \beta} \cos (k \tau), & k>0, \\ -\sqrt{2 / \beta} \sin (k \tau), & k<0, \\ \sqrt{1 / \beta}, & k=0\end{cases}
$$

is a base of the space $\mathcal{E}$. Here

$$
k \in \mathcal{K}=\{k=(2 \pi / \beta) \kappa \mid \kappa \in \mathbb{Z}\}
$$


are the so-called Matsubara frequencies. Let $P_{k}, k \in \mathcal{K}$ be the orthogonal projector on $e_{k}$. By $S$ we denote the linear operator $S: \mathcal{E} \rightarrow \mathcal{E}$, given by

$$
S=\sum_{k \in \mathcal{K}} \frac{1}{m k^{2}+a} P_{k}
$$

where $m$ and $a$ are the same as in (1.3). For this operator,

$$
S e_{k}=s_{k} e_{k}, \quad s_{k} \stackrel{\text { def }}{=} \frac{1}{m k^{2}+a}, \quad \operatorname{trace}(S)=\sum_{k \in \mathcal{K}} s_{k}<\infty .
$$

In the construction of the Euclidean Gibbs measures the central role is played by the symmetric Gaussian measure $\chi$, determined by its Fourier transformation

$$
\int_{\mathcal{E}} \exp \left(\mathrm{i}(x, y)_{\mathcal{E}}\right) \mathrm{d} \chi(y)=\exp \left\{(S x, x)_{\mathcal{E}} / 2\right\}
$$

Hence, (2.38) is the covariance operator for the measure $\chi$ and

$$
\int_{\mathcal{E}} y(\tau) y\left(\tau^{\prime}\right) \mathrm{d} \chi(y)=S\left(\tau, \tau^{\prime}\right) \stackrel{\text { def }}{=} \sum_{k \in \mathcal{K}} s_{k} e_{k}(\tau) e_{k}\left(\tau^{\prime}\right) .
$$

A complete account of the properties of $\chi$ is given in subsection 2.2 of [22]. It appears, that this measure is concentrated on a subset of the space $\mathcal{E}$. Set

$$
\mathcal{C}=\{x:[0, \beta] \rightarrow \mathbb{R} \mid x \text { is continuous and } x(0)=x(\beta)\} .
$$

The elements of $\mathcal{C}$ are called continuous loops. They are square integrable on $[0, \beta]$, hence, $\mathcal{C} \subset \mathcal{E}$. At the same time, the set $\mathcal{C}$ may be equipped with its own norm

$$
\|x\|_{\mathcal{C}}=\sup _{\tau \in[0, \beta]}|x(\tau)|
$$

which turns it into a Banach space. The measure $\chi$ is concentrated on this space, i.e., $\chi(\mathcal{C})=1$. This fact, together with some properties of the spaces $\mathcal{E}$ and $\mathcal{C}$, allow us also to consider $\chi$ as a measure on the Banach space $\mathcal{C}$. Given a box $\Lambda$, we set

$$
\mathcal{C}_{\Lambda}=\left\{x_{\Lambda}=\left(x_{\ell}\right)_{\ell \in \Lambda} \mid x_{\ell} \in \mathcal{C}\right\}
$$

which is the set of vectors with the components labelled by the elements of $\Lambda$, each component being a continuous loop. This set is equipped with the norm

$$
\left\|x_{\Lambda}\right\|_{\mathcal{C}_{\Lambda}}=\sup _{\ell \in \Lambda}\left\|x_{\ell}\right\|_{\mathcal{C}}
$$

which turns it into a Banach space. Now we set

$$
\mathrm{d} \chi_{\Lambda}\left(x_{\Lambda}\right)=\prod_{\ell \in \Lambda} \mathrm{d} \chi\left(x_{\ell}\right)
$$


which is a symmetric Gaussian measure on the Banach space (2.44). The measure $\chi$ describes an isolated harmonic oscillator of mass $m$ and rigidity $a$. Its two-point Matsubara function corresponding to the displacement operators is given by (2.41). The measure (2.45) describes a system of non-interacting harmonic oscillators in $\Lambda$. In order to also take into account the inter-particle interaction and the anharmonicity we introduce the energy functionals

$$
\begin{aligned}
& E_{\Lambda}^{V}\left(x_{\Lambda} \mid 0\right)=-\frac{1}{2} \sum_{\ell, \ell^{\prime} \in \Lambda} J_{\ell \ell^{\prime}} \int_{0}^{\beta} x_{\ell}(\tau) x_{\ell^{\prime}}(\tau) \mathrm{d} \tau+\sum_{\ell \in \Lambda} \int_{0}^{\beta} V\left(x_{\ell}(\tau)\right) \mathrm{d} \tau, \\
& E_{\Lambda}^{V}\left(x_{\Lambda} \mid p\right)=-\frac{1}{2} \sum_{\ell, \ell^{\prime} \in \Lambda} J_{\ell \ell^{\prime}}^{\Lambda} \int_{0}^{\beta} x_{\ell}(\tau) x_{\ell^{\prime}}(\tau) \mathrm{d} \tau+\sum_{\ell \in \Lambda} \int_{0}^{\beta} V\left(x_{\ell}(\tau)\right) \mathrm{d} \tau,
\end{aligned}
$$

which are continuous maps from the Banach space $\mathcal{C}_{\Lambda}$ into $\mathbb{R}$. Both are below bounded in view of (1.4). Set

$$
\begin{aligned}
\mathrm{d} \nu_{\Lambda}\left(x_{\Lambda} \mid 0\right) & =\frac{1}{Z_{\beta, \Lambda}(0)} \exp \left(-E_{\Lambda}^{V}\left(x_{\Lambda} \mid 0\right)\right) \mathrm{d} \chi_{\Lambda}\left(x_{\Lambda}\right), \\
\mathrm{d} \nu_{\Lambda}\left(x_{\Lambda} \mid p\right) & =\frac{1}{Z_{\beta, \Lambda}(p)} \exp \left(-E_{\Lambda}^{V}\left(x_{\Lambda} \mid p\right)\right) \mathrm{d} \chi_{\Lambda}\left(x_{\Lambda}\right),
\end{aligned}
$$

where

$$
\begin{aligned}
& Z_{\beta, \Lambda}(0)=\int_{\mathcal{C}_{\Lambda}} \exp \left(-E_{\Lambda}^{V}\left(x_{\Lambda} \mid 0\right)\right) \mathrm{d} \chi_{\Lambda}\left(x_{\Lambda}\right), \\
& Z_{\beta, \Lambda}(p)=\int_{\mathcal{C}_{\Lambda}} \exp \left(-E_{\Lambda}^{V}\left(x_{\Lambda} \mid p\right)\right) \mathrm{d} \chi_{\Lambda}\left(x_{\Lambda}\right),
\end{aligned}
$$

are the normalizing constants the role of which is to assure the measures (2.47) to be probabilistic. The crux of the Euclidean approach is contained in the following statement.

Proposition 7 The normalizing constants (2.48) coincide with the corresponding local partition functions (2.11). Moreover, the Matsubara functions (2.23), (2.24) corresponding to multiplication operators $F_{1}, \ldots F_{n}$ have the representations

$$
\begin{aligned}
\Gamma_{F_{1}, \ldots, F_{n}}^{0, \beta, \Lambda}\left(\tau_{1}, \ldots, \tau_{n}\right) & =\int_{\mathcal{C}_{\Lambda}} F_{1}\left(x_{\Lambda}\left(\tau_{1}\right)\right) \cdots F_{n}\left(x_{\Lambda}\left(\tau_{n}\right)\right) \mathrm{d} \nu_{\Lambda}\left(x_{\Lambda} \mid 0\right), \\
\Gamma_{F_{1}, \ldots, F_{n}}^{p, \beta, \Lambda}\left(\tau_{1}, \ldots, \tau_{n}\right) & =\int_{\mathcal{C}_{\Lambda}} F_{1}\left(x_{\Lambda}\left(\tau_{1}\right)\right) \cdots F_{n}\left(x_{\Lambda}\left(\tau_{n}\right)\right) \mathrm{d} \nu_{\Lambda}\left(x_{\Lambda} \mid p\right) .
\end{aligned}
$$

For more details, including the proof, the reader is referred to the article [12] and to the references in [22]. Since, by Corollary 6, the functions (2.49) corresponding to multiplication operators by continuous functions $F_{1}, \ldots, F_{n}$ completely determine the local Gibbs states, the measures (2.47) also possess this property. In what follows, we have established the one-to-one correspondence between the local Gibbs states (2.14) and the probability measures (2.47). This is the reason to call them local 
Euclidean Gibbs measures. This correspondence allows us to translate the description of the model from the "algebraic" language to the language of probability measures, which paves the way to employing a plenty of powerful techniques developed in stochastic analysis and classical statistical physics [33].

\subsection{Infinite-volume Euclidean Gibbs states}

As was mentioned above, there is no way of getting the group of time maps describing infinite-volume dynamics of our model, and hence, to define its infinitevolume Gibbs states as $\beta$-KMS states. Therefore, the only way of defining such states is to use the language of probability measures. To this end we introduce conditional local Gibbs measures. To make the things as easy as we can from now on we suppose that the interaction potential is of finite range. This means that there exists $R>0$ such that $J_{\ell \ell^{\prime}}=0$ whenever $\left|\ell-\ell^{\prime}\right|>R$. A typical example is the nearest-neighbor interaction. Consider

$$
\Omega=\left\{x=\left(x_{\ell}\right)_{\ell \in \mathbb{L}} \mid x_{\ell} \in \mathcal{C}\right\} .
$$

This set consists of vectors $x$ - configurations - which have infinitely many components labelled by lattice sites running through the lattice $\mathbb{L}$, each component being a continuous loop. The Euclidean Gibbs states we are going to construct are probability measures on $\Omega$. We do not give a complete description of these measures. The reader who would desire to get into the details is referred to $[12,22]$. Given $\xi \in \Omega$, we set

$$
\begin{aligned}
\mathrm{d} \nu_{\Lambda}\left(x_{\Lambda} \mid \xi\right) & =\frac{1}{Z_{\beta, \Lambda}(\xi)} \exp \left(-E_{\Lambda}^{V}\left(x_{\Lambda} \mid \xi\right)\right) \mathrm{d} \chi_{\Lambda}\left(x_{\Lambda}\right), \\
Z_{\beta, \Lambda}(\xi) & =\int_{\mathcal{C}_{\Lambda}} \exp \left(-E_{\Lambda}^{V}\left(x_{\Lambda} \mid \xi\right)\right) \mathrm{d} \chi_{\Lambda}\left(x_{\Lambda}\right),
\end{aligned}
$$

where

$$
E_{\Lambda}^{V}\left(x_{\Lambda} \mid \xi\right) \stackrel{\text { def }}{=} E_{\Lambda}^{V}\left(x_{\Lambda} \mid 0\right)-\sum_{\ell \in \Lambda, \ell^{\prime} \in \Lambda^{c}} J_{\ell \ell^{\prime}} \int_{0}^{\beta} x_{\ell}(\tau) \xi_{\ell^{\prime}}(\tau) \mathrm{d} \tau,
$$

$\Lambda^{c}=\mathbb{L} \backslash \Lambda$ is the complement of $\Lambda$ and the energy functional $E_{\Lambda}^{V}\left(x_{\Lambda} \mid 0\right)$ is given by (2.46). The energy functional (2.52), along with the interaction inside $\Lambda$ also describes the interaction of the particles in $\Lambda$ with the configuration $\xi$ fixed outside $\Lambda$. The measure (2.51) is a conditional local Euclidean Gibbs measure, conditioned by the configuration $\xi$ fixed outside $\Lambda$. Given $\ell \in \Lambda$, let $\mathrm{d} \delta_{\xi_{\ell}}\left(x_{\ell}\right)$ be the Dirac measure concentrated at $\xi_{\ell}$, i.e.,

$$
\mathrm{d} \delta_{\xi_{\ell}}\left(x_{\ell}\right) \stackrel{\text { def }}{=} \delta\left(x_{\ell}-\xi_{\ell}\right) \mathrm{d} x_{\ell} .
$$

Here the $\delta$ on the right-hand side is "the usual Dirac delta-function", which, along with $\mathrm{d} x_{\ell}$, is only a heuristic expression. However, the left-hand side of (2.53) is a well-defined probability measure on $\mathcal{C}$. Furthermore, we set

$$
\mathrm{d} \pi_{\Lambda}(x \mid \xi)=\mathrm{d} \nu\left(x_{\Lambda} \mid \xi\right) \prod_{\ell \in \Lambda^{c}} \mathrm{~d} \delta_{\xi_{\ell}}\left(x_{\ell}\right)
$$


In contrast to the measure (2.51) the above $\pi_{\Lambda}$ is a measure on the space $\Omega$. It defines a probability distribution on the space of configurations on the whole lattice, but it takes nonzero values only for those configurations which outside $\Lambda$ coincide with the fixed configuration $\xi$. Let $\mu$ be a probability measure of $\Omega$. Then

$$
\mathrm{d} \tilde{\mu}(x)=\int_{\Omega} \mathrm{d} \pi_{\Lambda}(x \mid \xi) \mathrm{d} \mu(\xi),
$$

is again a probability measure on $\Omega$. The difference between the latter one and the measure (2.54) is as follows. Both measures are built up with the Hamiltonian $H_{\Lambda}(0)$, but the former measure describes the interaction of the particles in $\Lambda$ with a fixed configuration outside $\Lambda$ whereas in the latter measure, boundary conditions are averaged with the measure $\mu$. This means that it defines the probability distribution of configurations in $\Lambda$ interacting with configurations outside $\Lambda$, distributed according to the measure $\mu$. If $\tilde{\mu}=\mu$, both configurations - inside and outside $\Lambda-$ are in equilibrium. These arguments lead to the following definition.

Definition 8 A probability measure $\mu$ on the set of all configurations $\Omega$ is said to be a Euclidean Gibbs measure at temperature $\beta^{-1}$ if, for any box $\Lambda$,

$$
\mathrm{d} \mu(x)=\int_{\Omega} \mathrm{d} \pi_{\Lambda}(x \mid \xi) \mathrm{d} \mu(\xi) .
$$

The set of all Euclidean Gibbs measures is denoted by $\mathcal{G}_{\beta}$.

In view of the above arguments (2.56) is called the equilibrium equation or the Dobrushin-Lanford-Ruelle (DLR) equation. More on this subject one may find in [33]. In order to exclude configurations with no physical meaning certain restrictions on $\Omega$ are imposed. We recall that for a given configuration $\xi \in \Omega$, its components $\xi_{\ell}$ are continuous loops, hence, they are elements of the Hilbert space $\mathcal{E}$. Set

$$
\Omega^{\mathrm{t}}=\left\{\xi \in \Omega \mid \forall \alpha>0: \sum_{\ell \in \mathbb{L}}\left\|\xi_{\ell}\right\|_{\mathcal{E}} \mathrm{e}^{-\alpha|\ell|}<\infty\right\} .
$$

The elements of this set are called tempered configurations, which means that their norms grow with $|\ell| \rightarrow+\infty$ not very rapidly. A Euclidean Gibbs measure $\mu$ is called tempered if

$$
\mu\left(\Omega^{\mathrm{t}}\right)=1,
$$

i.e., it is concentrated on the set of tempered configurations. The set of all tempered Euclidean Gibbs measures is denoted by $\mathcal{G}_{\beta}^{\mathrm{t}}$. Like in the case of KMS states, a convex combination (2.31) of tempered Euclidean Gibbs measures is again a tempered Euclidean Gibbs measure. A priori it is not clear whether the sets $\mathcal{G}_{\beta}^{\mathrm{t}}$, or even $\mathcal{G}_{\beta}$, contain at least one element. A complete answer to this question was found only recently in [34], where it was proven that, for the model considered, the set $\mathcal{G}_{\beta}^{\mathrm{t}}$ is nonempty and a number of properties of its elements were established. The elements of $\mathcal{G}_{\beta}^{\mathrm{t}}$ which are not the mixtures of its other elements are called extreme elements of $\mathcal{G}_{\beta}^{\mathrm{t}}$. If the latter set consists of just one element, it is extreme. Only extreme elements correspond to physical phases. 


\section{Quantum stabilization}

\subsection{Quantum effects}

The question as to whether or not the ordering in the system of light particles, which we describe by the Hamiltonian (1.2), (1.3), is indeed responsible for the macroscopic displacements of ionic complexes may be answered by means of experiments in which the properties of the light particles are changed. In the case of the KDP-type compounds there were performed two types of experiments changing the properties of hydrogen bonds. In the first group of experiments a part of hydrogen nuclei, i.e., protons, were replaced by deuterium nuclei, which have the same electric charge but approximately two times bigger mass. Among the effects there was a substantial increase of the Curie temperature, depending on the concentration of deuterons (the so-called isotopic effect). Another group of experiments dealt with changing the potential energy of the crystalline field by applying high hydrostatic pressure, which makes the minima of the double wells closer to each other and increases the tunnelling of protons. As a consequence, the Curie temperature decreases. These results are described in the book [35], see the table on page 188, see also [36] for further development. Clearly, the isotopic effect and the effect of pressure on the phase transition are of the quantum mechanical origin. Thus, the question as to whether the model describes, at least qualitatively, such quantum effects becomes very important - a positive answer would confirm the adequacy of the model. First the problem of quantum effects in models like (1.2), (1.3) was discussed in the pioneering paper [37]. In that paper arguments in favor of quantum effects were obtained by means of the Bogoliubov inequalities. Later some exactly soluble models of interacting quantum anharmonic oscillators manifesting quantum effects were proposed $[38,39]$. The first rigorous proof that the long range order in the model (1.2), (1.3) may be suppressed at all temperatures in the limit $m \rightarrow 0$ was given in [40]. At the same time, no explanation of the effect of pressure was given and the quantum mechanical origin of this effect has remained unclear. In this article in the framework of the Euclidean approach outlined above we propose a mathematically rigorous description of the mechanism of quantum stabilization, which explains both these effects and the role of the anharmonism. Its physical essence may be explained as follows. If the tunnelling between the wells gets intensive (smaller mass or closer minima), the particle "forgets" about the details of the potential energy in the vicinity of the origin (i.e., of the point $q_{\ell}=0$ ), including the instability of its equilibrium at this point. It oscillates as if this equilibrium were stable. If the potential energy grows at infinity faster than $\xi^{2}$, the oscillations become more and more rigid as $m \rightarrow 0$. When this effective rigidity exceeds the total force $\sum_{\ell^{\prime}} J_{\ell \ell^{\prime}}$, the system gets stable at all temperatures, which means, in particular, that the spatial decay of the two-point correlation function is exponential. This results in preventing the model from any critical point anomaly, all the more from the long range ordering. In a similar model, the exponential decay of correlations for small values of the mass was proven in [41]. The proof was based on cluster expansions. No stability arguments were used. A deeper result of such stabilization turns out to 
be the uniqueness of the tempered Euclidean Gibbs measures, which was proven in $[21,23]$.

\subsection{Decay of correlations and consequences}

Given a box $\Lambda$ and $\ell, \ell^{\prime} \in \Lambda$, we set

$$
\begin{aligned}
& K_{\ell \ell^{\prime}}\left(\tau, \tau^{\prime} \mid \Lambda, 0\right)=\Gamma_{q_{\ell}, q_{\ell^{\prime}}}^{0, \beta, \Lambda}\left(\tau, \tau^{\prime}\right), \\
& K_{\ell \ell^{\prime}}\left(\tau, \tau^{\prime} \mid \Lambda, p\right)=\Gamma_{q_{\ell}, q_{\ell^{\prime}}}^{p, \beta, \Lambda}\left(\tau, \tau^{\prime}\right),
\end{aligned}
$$

where $\tau, \tau^{\prime} \in[0, \beta]$ and the Matsubara functions are defined by $(2.23),(2.24)$. Then by $(2.49)$,

$$
\begin{aligned}
K_{\ell \ell^{\prime}}\left(\tau, \tau^{\prime} \mid \Lambda, 0\right) & =\int_{\mathcal{C}_{\Lambda}} x_{\ell}(\tau) x_{\ell^{\prime}}\left(\tau^{\prime}\right) \mathrm{d} \nu_{\Lambda}\left(x_{\Lambda} \mid 0\right), \\
K_{\ell \ell^{\prime}}\left(\tau, \tau^{\prime} \mid \Lambda, p\right) & =\int_{\mathcal{C}_{\Lambda}} x_{\ell}(\tau) x_{\ell^{\prime}}\left(\tau^{\prime}\right) \mathrm{d} \nu_{\Lambda}\left(x_{\Lambda} \mid p\right) .
\end{aligned}
$$

We recall that the interaction potential in our model has a finite range. Set

$$
\hat{J}(\Lambda)=\sum_{\ell^{\prime} \in \mathbb{L}} J_{\ell \ell^{\prime}} \exp \left(\mathrm{i}\left(\lambda, \ell-\ell^{\prime}\right)\right), \quad \lambda \in(-\pi, \pi]^{d},
$$

and

$$
I(\lambda)=\hat{J}(0)-\hat{J}(\lambda)
$$

We suppose that the anharmonic potential $V$ is an even differentiable function satisfying (1.4). Moreover, we suppose that the function $v(t), t \in[0,+\infty)$, determined by the relation

$$
V(\xi)=v\left(\xi^{2}\right), \quad \xi \in \mathbb{R},
$$

which holds since $V$ is even, is such that

$$
\frac{\mathrm{d}^{2} v(t)}{\mathrm{d} t^{2}} \geqslant 0, \quad \text { for all } t \in[0,+\infty)
$$

This implies that $V(\xi)$ grows faster than $\xi^{2}$ as $\xi \rightarrow \infty$. The polynomial (1.5) with nonnegative $b_{2}, \ldots, b_{r-1}$ certainly satisfies (3.5). Under these assumptions the Hamiltonian $H_{\ell}$ has a pure point spectrum consisting of simple eigenvalues $E_{n}, n=0,1, \ldots$ (see e.g., [30]). Thus, we may set

$$
\Delta=\min _{n \in \mathbb{N}}\left\{E_{n}-E_{n-1}\right\}
$$

For $d \geqslant 3$, the long range order in the model is possible if $V$ has two deep wells separated by a sufficiently high barrier, see $[15,16]$. In this case $\Delta$ is the frequency of the tunnelling between the wells. Thus, the long range order appears if $\Delta$ is small. If it is big enough, the correlation functions (3.2) decay exponentially, which is described by the following statement. 
Theorem 9 Let the conditions

$$
m \Delta^{2}>\hat{J}(0)
$$

and (3.5) be satisfied. Then, for any $\beta>0$ and any box $\Lambda$, for all $\ell, \ell^{\prime} \in \Lambda$ and $\tau, \tau^{\prime} \in[0, \beta]$, the following estimates hold

$$
\begin{aligned}
0 & \leqslant K_{\ell \ell^{\prime}}\left(\tau, \tau^{\prime} \mid \Lambda, 0\right) \\
& \leqslant \frac{1}{\beta(2 \pi)^{d}} \sum_{k \in \mathcal{K}} \int_{(-\pi, \pi]^{d}} \frac{\exp \left(\mathrm{i} k\left(\tau-\tau^{\prime}\right)+\mathrm{i}\left(\lambda, \ell-\ell^{\prime}\right)\right)}{\Xi_{\beta}(k)+I(\lambda)} \mathrm{d} \lambda
\end{aligned}
$$

where $\Xi_{\beta}(k)$ is such that uniformly in $\beta$

$$
\Xi_{\beta}(k) \geqslant m \Delta^{2}+m k^{2}-\hat{J}(0) .
$$

Let us fix $\ell, \ell^{\prime} \in \mathbb{L}$ and $\tau, \tau^{\prime} \in[0, \beta]$. Then one can find $L_{0} \in \mathbb{N}$ such that $\ell, \ell^{\prime}$ belong to the box $\Lambda_{L_{0}},(2.6)$, and hence, to all elements of the sequence of boxes $\mathcal{L}=\left\{\Lambda_{L}\right\}_{L \geqslant L_{0}}$ The next statement is a corollary of Theorem 9.

Theorem 10 Under the conditions of Theorem 9 the limit

$$
\lim _{L \rightarrow+\infty} K_{\ell \ell^{\prime}}\left(\tau, \tau^{\prime} \mid \Lambda_{L}, 0\right) \stackrel{\text { def }}{=} K_{\ell \ell^{\prime}}\left(\tau, \tau^{\prime} \mid 0\right)
$$

exists, it obeys (3.8).

The proof of both theorems will be performed in several steps in the concluding subsection. Now we describe their certain corollaries.

One may show (see [25-27]) that under the condition $a+V^{\prime \prime}(0)>\hat{J}(0)$ the Euclidean Gibbs state is unique; hence, no phase transitions and no critical points occur. However, for $d \geqslant 3$ and $a+V^{\prime \prime}(0)<\hat{J}(0)$, a structural phase transition becomes possible if the mass $m$ and the inverse temperature $\beta$ are big enough (see [16] for the general case and [2,3] for the case of $r=2$ ). This phase transition is of displacive type if $0 \leqslant a+V^{\prime \prime}(0)<\hat{J}(0)$, and it is of order-disorder type if $a+V^{\prime \prime}(0)<0$ (see $[4,35,37]$ ). In both cases the instability which leads to the phase transition causes the appearance of soft-mode collective excitations (see page 11-18 of $[35])$.

Corollary 11 Under the conditions of Theorem 9 the soft-mode excitations are absent (suppressed) at all temperatures.

Proof: By (3.9), the denominator of the expression on the right-hand side of (3.8) is positive for all $\lambda \in(-\pi, \pi]^{d}$ and $k \in \mathcal{K}$ if the condition (3.7) holds. Thus, the energy of elementary excitations $\epsilon(\lambda)$ has a positive gap $\epsilon(0) \geqslant m \Delta^{2}-\hat{J}(0)$, hence, no soft-mode phenomena occur.

Set

$$
\varpi_{\Lambda}=\frac{1}{|\Lambda|} \sum_{\ell, \ell^{\prime} \in \Lambda} \int_{0}^{\beta} K_{\ell \ell^{\prime}}(0, \tau \mid \Lambda, 0) \mathrm{d} \tau,
$$

which is the static susceptibility in the box $\Lambda$. If at a given $\beta$, the sequence $\left\{\varpi_{\Lambda_{L}}\right\}_{L \in \mathbb{N}}$ is bounded, then no critical point anomaly at this $\beta$ occurs. 
Corollary 12 Under the conditions of Theorem 9 the sequence $\left\{\varpi_{\Lambda_{L}}\right\}_{L \in \mathbb{N}}$ is convergent at all temperatures to a certain $\varpi_{\infty}<\infty$.

The proof will be performed in the concluding subsection.

Let us look at the above statements from the following point of view. In the harmonic case the Hamiltonian (1.2), (1.3) may be rewritten in the form

$$
H^{\text {har }}=\frac{1}{2 m} \sum_{\ell} p_{\ell}^{2}+\frac{1}{2} \sum_{\ell, \ell^{\prime}}\left[a \delta_{\ell \ell^{\prime}}-J_{\ell \ell^{\prime}}\right] q_{\ell} q_{\ell^{\prime}}
$$

where $\delta_{\ell \ell^{\prime}}$ is the Kronecker delta. The system described by this Hamiltonian is stable if $a>\hat{J}(0)$. This condition is also applicable to the classical analog of (3.12). In the quantum case one may write $a=m \Delta_{\text {har }}^{2}$, where $\Delta_{\text {har }}$ is defined as in (3.6) but with the eigenvalues of the Hamiltonian $H_{\ell}^{\text {har }}=\left(p_{\ell}^{2} / 2 m\right)+(a / 2) q_{\ell}^{2}$. Thus, the system (3.12) is stable if

$$
m \Delta_{\text {har }}^{2}>\hat{J}(0) \text {. }
$$

Under this condition the infinite-volume Euclidean Gibbs state of (3.12) exists and is unique at all temperatures. Moreover, the corresponding infinite volume correlation function can be calculated explicitly. It is

$$
K_{\ell \ell^{\prime}}^{\mathrm{har}}\left(\tau, \tau^{\prime} \mid 0\right)=\frac{1}{\beta(2 \pi)^{d}} \sum_{k \in \mathcal{K}} \int_{(-\pi, \pi]^{d}} \frac{\exp \left(\mathrm{i} k\left(\tau-\tau^{\prime}\right)+\mathrm{i}\left(\lambda, \ell-\ell^{\prime}\right)\right)}{m \Delta_{\text {har }}^{2}+m k^{2}-\hat{J}(0)+I(\lambda)} \mathrm{d} \lambda .
$$

Comparing the conditions (3.13) and (3.7) one comes to the following conclusion. The parameter $m \Delta^{2}$ may be considered as the measure of the due-to-quantum-effects rigidity of the anharmonic oscillator which has an unstable equilibrium at $q_{\ell}=0$. One can show that $m \Delta^{2} \rightarrow 0$ as $m \rightarrow+\infty$. For $V$ being of the form (1.5), it is a continuous function of $m$ such that $m \Delta^{2} \sim C m^{-(r-1) /(r+1)}$ as $m \rightarrow 0$ (see $[19,22]$ ). Thus, in the quasi-classical limit $m \rightarrow+\infty$ (see [20,22]) the quantum rigidity $m \Delta^{2}$ disappears due to the instability at $q_{\ell}=0$. On the other hand, this rigidity becomes large either if $m$ gets small or if the tunnelling $\Delta$ gets large. If the condition (3.7) is satisfied, the spatial decay of the correlation function (3.10) is not less than it is for its harmonic analog (3.14). It is exponential if $I(\lambda) \sim j|\lambda|^{2}$ as $\lambda \rightarrow 0$ (see e.g., claim (c) of Proposition 7.2.1 page 162 of [42]), which is obviously the case for interaction potentials of finite range.

\subsection{The proof of theorems 9,10 and corollary 12}

For a one-point box $\Lambda=\{\ell\}$, we have

$$
\begin{aligned}
K_{\ell \ell}\left(\tau, \tau^{\prime} \mid\{\ell\}, 0\right) & =\frac{1}{Z_{\ell}} \operatorname{trace}\left\{q_{\ell} \exp \left(-\left(\tau-\tau^{\prime}\right) H_{\ell}\right) q_{\ell} \exp \left(-\left(\beta-\tau+\tau^{\prime}\right) H_{\ell}\right)\right\} \\
Z_{\ell} & =\operatorname{trace}\left\{\exp \left(-\beta H_{\ell}\right)\right\} .
\end{aligned}
$$

Set

$$
U(k)=\int_{0}^{\beta} K_{\ell \ell}\left(\tau, \tau^{\prime} \mid\{\ell\}, 0\right) \cos \left[k\left(\tau-\tau^{\prime}\right)\right] \mathrm{d} \tau^{\prime}, \quad k \in \mathcal{K} .
$$

Obviously, $U(k)$ does not depend on $\ell$ and $\tau$. 
Lemma 13 For every $\beta>0$ and $k \in \mathcal{K}$,

$$
0 \leqslant U(k) \leqslant \frac{1}{m\left(\Delta^{2}+k^{2}\right)}
$$

Proof: Let $\psi_{n}, n=0,1,2, \ldots$ be the eigenfunction of the Hamiltonian $H_{\ell}$ which corresponds to the eigenvalue $E_{n}$. Set

$$
Q_{n n^{\prime}}=\left\langle\psi_{n}\left|q_{\ell}\right| \psi_{n^{\prime}}\right\rangle
$$

Clearly, $Q_{n n^{\prime}}=\bar{Q}_{n^{\prime} n}$ does not depend on $\ell$ and $Q_{n n}=0$. By (3.15), (3.16), one has

$$
\begin{aligned}
U(k) & =\frac{1}{Z_{\ell}} \sum_{n, n^{\prime}=0}^{\infty}\left|Q_{n n^{\prime}}\right|^{2} \frac{E_{n}-E_{n^{\prime}}}{\left[E_{n}-E_{n^{\prime}}\right]^{2}+k^{2}}\left\{\exp \left(-\beta E_{n^{\prime}}\right)-\exp \left(-\beta E_{n}\right)\right\} \\
& \leqslant \frac{1}{\Delta^{2}+k^{2}} \cdot \frac{1}{Z_{\ell}} \sum_{n, n^{\prime}=0}^{\infty}\left|Q_{n n^{\prime}}\right|^{2}\left[E_{n}-E_{n^{\prime}}\right]\left\{\exp \left(-\beta E_{n^{\prime}}\right)-\exp \left(-\beta E_{n}\right)\right\} \\
& =\frac{1}{\Delta^{2}+k^{2}} \cdot \frac{1}{Z_{\ell}} \operatorname{trace}\left\{\left[q_{\ell},\left[H_{\ell}, q_{\ell}\right]\right] \exp \left(-\beta H_{\ell}\right)\right\} \\
& =\frac{1}{m\left(\Delta^{2}+k^{2}\right)},
\end{aligned}
$$

where to get the latter equality we have used (1.1), (1.3) and (3.15). The lower bound in (3.17) follows from the first two lines of the above formula.

Along with $(2.47)$ we use the following measure. For $t \in[0,1]$, we set

$$
\begin{aligned}
\mathrm{d} \nu_{\Lambda}^{(t)}\left(x_{\Lambda} \mid p\right)= & \frac{1}{Z_{\beta, \Lambda}^{(t)}(p)} \exp \left\{\frac{t}{2} \sum_{\ell, \ell^{\prime} \in \Lambda} J_{\ell \ell^{\prime}}^{\Lambda} \int_{0}^{\beta} x_{\ell}(\tau) x_{\ell^{\prime}}(\tau) \mathrm{d} \tau\right. \\
& \left.-\sum_{\ell \in \Lambda} \int_{0}^{\beta} V\left(x_{\ell}(\tau)\right) \mathrm{d} \tau\right\} \mathrm{d} \chi_{\Lambda}\left(x_{\Lambda}\right),
\end{aligned}
$$

where

$$
\begin{aligned}
Z_{\beta, \Lambda}^{(t)}(p)= & \int_{\mathcal{C}_{\Lambda}} \exp \left\{\frac{t}{2} \sum_{\ell, \ell^{\prime} \in \Lambda} J_{\ell \ell^{\prime}}^{\Lambda} \int_{0}^{\beta} x_{\ell}(\tau) x_{\ell^{\prime}}(\tau) \mathrm{d} \tau\right. \\
& \left.-\sum_{\ell \in \Lambda} \int_{0}^{\beta} V\left(x_{\ell}(\tau)\right) \mathrm{d} \tau\right\} \mathrm{d} \chi_{\Lambda}\left(x_{\Lambda}\right),
\end{aligned}
$$

By means of the Gaussian upper bound estimate (see Theorem 6.4 in [22]), one can show that $Z_{\beta, \Lambda}^{(t)}(p)$, as well as similar integrals, can be analytically continued to entire functions of $t \in \mathbb{C}$. Clearly, $Z_{\beta, \Lambda}^{(t)}(p)$ is positive for $t \in[0,1]$. Set

$$
\begin{aligned}
S_{\ell_{1} \ell_{2} \ell_{3} \ell_{4}}\left(\tau_{1}, \tau_{2}, \tau_{3}, \tau_{4} \mid t\right) & =\int_{\mathcal{C}_{\Lambda}} x_{\ell_{1}}\left(\tau_{1}\right) x_{\ell_{2}}\left(\tau_{2}\right) x_{\ell_{3}}\left(\tau_{3}\right) x_{\ell_{4}}\left(\tau_{4}\right) \mathrm{d} \nu_{\Lambda}^{(t)}\left(x_{\Lambda} \mid p\right), \\
X_{\ell \ell^{\prime}}\left(\tau, \tau^{\prime} \mid t\right) & =\int_{\mathcal{C}_{\Lambda}} x_{\ell}(\tau) x_{\ell^{\prime}}\left(\tau^{\prime}\right) \mathrm{d} \nu_{\Lambda}^{(t)}\left(x_{\Lambda} \mid p\right)
\end{aligned}
$$


and

$$
\begin{aligned}
& R_{\ell_{1} \ell_{2} \ell_{3} \ell_{4}}\left(\tau_{1}, \tau_{2}, \tau_{3}, \tau_{4} \mid t\right)= \\
&= S_{\ell_{1} \ell_{2} \ell_{3} \ell_{4}}\left(\tau_{1}, \tau_{2}, \tau_{3}, \tau_{4} \mid t\right)-X_{\ell_{1} \ell_{2}}\left(\tau_{1}, \tau_{2} \mid t\right) X_{\ell_{3} \ell_{4}}\left(\tau_{3}, \tau_{4} \mid t\right) \\
& \quad-X_{\ell_{1} \ell_{3}}\left(\tau_{1}, \tau_{3} \mid t\right) X_{\ell_{2} \ell_{4}}\left(\tau_{2}, \tau_{4} \mid t\right)-X_{\ell_{1} \ell_{4}}\left(\tau_{1}, \tau_{4} \mid t\right) X_{\ell_{2} \ell_{3}}\left(\tau_{2}, \tau_{3} \mid t\right) .
\end{aligned}
$$

By the Lebowitz inequality (see Theorem 6.3 in [22]), which holds for the moments of the measure (3.18) with the anharmonicity potential $V$ satisfying the condition (3.5), one has

$$
R_{\ell_{1} \ell_{2} \ell_{3} \ell_{4}}\left(\tau_{1}, \tau_{2}, \tau_{3}, \tau_{4} \mid t\right) \leqslant 0
$$

which holds for all $\ell_{1}, \ldots, \ell_{4} \in \Lambda, \tau_{1}, \ldots, \tau_{4} \in[0, \beta]$ and $t \in[0,1]$. As it has been mentioned above, $X_{\ell \ell^{\prime}}\left(\tau, \tau^{\prime} \mid t\right)$ is a differentiable function of $t \in(0,1)$ for any fixed $\ell, \ell^{\prime} \in \Lambda$ and $\tau, \tau^{\prime} \in[0, \beta]$. By direct calculation,

$$
\begin{aligned}
\frac{\partial}{\partial t} X_{\ell \ell^{\prime}}\left(\tau, \tau^{\prime} \mid t\right)= & \frac{1}{2} \sum_{\ell_{1}, \ell_{2} \in \Lambda} J_{\ell_{1} \ell_{2}}^{\Lambda} \int_{0}^{\beta} R_{\ell \ell^{\prime} \ell_{1} \ell_{2}}\left(\tau, \tau^{\prime}, \tau_{1}, \tau_{1} \mid t\right) \mathrm{d} \tau_{1} \\
& +\sum_{\ell_{1}, \ell_{2} \in \Lambda} J_{\ell_{1} \ell_{2}}^{\Lambda} \int_{0}^{\beta} X_{\ell \ell_{1}}\left(\tau, \tau_{1} \mid t\right) X_{\ell^{\prime} \ell_{2}}\left(\tau^{\prime}, \tau_{1} \mid t\right) \mathrm{d} \tau_{1} .
\end{aligned}
$$

This is an integro-differential equation subject to the following boundary conditions (see $(3.2),(3.18)-(3.20))$

$$
X_{\ell \ell^{\prime}}\left(\tau, \tau^{\prime} \mid 1\right)=K_{\ell \ell^{\prime}}\left(\tau, \tau^{\prime} \mid \Lambda, p\right) ; \quad X_{\ell \ell^{\prime}}\left(\tau, \tau^{\prime} \mid 0\right)=\delta_{\ell \ell^{\prime}} K_{\ell \ell}\left(\tau, \tau^{\prime} \mid\{\ell\}, 0\right)
$$

The solution of (3.23), (3.24) exists, is unique and has the form (3.20). We will compare it with the solution of the following problem

$$
\begin{aligned}
\frac{\partial}{\partial t} Y_{\ell \ell^{\prime}}\left(\tau, \tau^{\prime} \mid t\right) & =\frac{1}{2} \sum_{\ell_{1}, \ell_{2} \in \Lambda}\left[J_{\ell_{1} \ell_{2}}^{\Lambda}+\frac{\varepsilon}{|\Lambda|}\right] \int_{0}^{\beta} Y_{\ell \ell_{1}}\left(\tau, \tau_{1} \mid t\right) Y_{\ell^{\prime} \ell_{2}}\left(\tau^{\prime}, \tau_{1} \mid t\right) \mathrm{d} \tau_{1}, \\
Y_{\ell \ell^{\prime}}\left(\tau, \tau^{\prime} \mid 0\right) & =X_{\ell \ell^{\prime}}\left(\tau, \tau^{\prime} \mid 0\right)=\delta_{\ell \ell^{\prime}} K_{\ell \ell}\left(\tau, \tau^{\prime} \mid\{\ell\}, 0\right),
\end{aligned}
$$

where $\varepsilon \geqslant 0$ is a parameter.

Lemma 14 Let $U(k)$, defined by (3.16), and $\hat{J}(\lambda)$, defined by (3.3), obey the condition

$$
U(0) \hat{J}(0)<1 \text {. }
$$

Then there exists $\varepsilon_{0}>0$ such that, for all $\varepsilon \in\left[0, \varepsilon_{0}\right)$, the Cauchy problem (3.25) has a unique solution on $t \in[0,1]$, which has the form

$$
Y_{\ell \ell^{\prime}}\left(\tau, \tau^{\prime} \mid t\right)=\frac{1}{\beta|\Lambda|} \sum_{\lambda \in \Lambda_{*}} \sum_{k \in \mathcal{K}} \frac{\exp \left[\mathrm{i} k\left(\tau-\tau^{\prime}\right)+\mathrm{i}\left(\lambda, \ell-\ell^{\prime}\right)\right]}{[U(k)]^{-1}-t\left(\hat{J}^{\Lambda}(0)+\varepsilon \delta_{\lambda, 0}\right)+t I^{\Lambda}(\lambda)},
$$

where $\delta_{\lambda, 0}$ is the Kronecker delta, $I^{\Lambda}(\lambda)=\hat{J}^{\Lambda}(0)-\hat{J}^{\Lambda}(\lambda)$ and, for $\Lambda=\Lambda_{L}$ having the form (2.6)

$$
\Lambda_{*}=\left\{\lambda=\left(\lambda_{1}, \ldots, \lambda_{d}\right) \mid \lambda_{j}=-\pi+(\pi / L) n_{j}, \quad n_{j}=1, \ldots, 2 L, \quad j=1, \ldots, d\right\}
$$


Proof: By means of the Fourier transformation

$$
\begin{aligned}
Y_{\ell \ell^{\prime}}\left(\tau, \tau^{\prime} \mid t\right) & =\frac{1}{\beta|\Lambda|} \sum_{\lambda \in \Lambda_{*}} \sum_{k \in \mathcal{K}} \hat{Y}(k, \lambda \mid t) \exp \left[\mathrm{i} k\left(\tau-\tau^{\prime}\right)+\mathrm{i}\left(\lambda, \ell-\ell^{\prime}\right)\right], \\
\hat{Y}(k, \lambda \mid t) & =\sum_{\ell^{\prime} \in \Lambda} \int_{0}^{\beta} Y_{\ell \ell^{\prime}}\left(\tau, \tau^{\prime} \mid t\right) \exp \left[\mathrm{i} k\left(\tau-\tau^{\prime}\right)+\mathrm{i}\left(\lambda, \ell-\ell^{\prime}\right)\right] \mathrm{d} \tau^{\prime},
\end{aligned}
$$

we bring (3.25) into the form

$$
\frac{\partial}{\partial t} \hat{Y}(k, \lambda \mid t)=\left[\hat{J}^{\Lambda}(\lambda)+\varepsilon \delta_{\lambda, 0}\right][\hat{Y}(k, \lambda \mid t)]^{2}, \quad \hat{Y}(k, \lambda \mid 0)=U(k) .
$$

Clearly, $\hat{J}^{\Lambda}(\lambda) \leqslant \hat{J}^{\Lambda}(0) \leqslant \hat{J}(0)$ and $U(k) \leqslant U(0)$, thus, in view of (3.26), one finds $\varepsilon_{0}>0$ such that, for all $\varepsilon \in\left[0, \varepsilon_{0}\right)$, the following holds

$$
\left[\hat{J}^{\Lambda}(\lambda)+\varepsilon \delta_{\lambda, 0}\right] U(k)<1
$$

for all $\lambda \in \Lambda_{*}$ and $k \in \mathcal{K}$. Thus, the problem (3.29) may be solved explicitly, which via the Fourier transformation yields (3.27).

Given $\theta \in(0,1)$, we set

$$
Y_{\ell \ell^{\prime}}^{(\theta)}\left(\tau, \tau^{\prime} \mid t\right)=Y_{\ell \ell^{\prime}}\left(\tau, \tau^{\prime} \mid t+\theta\right), \quad t \in[0,1-\theta]
$$

Obviously, this function of $t$ obeys the equation (3.25) on $t \in[0,1-\theta]$ with the following initial condition

$$
Y_{\ell \ell^{\prime}}^{(\theta)}\left(\tau, \tau^{\prime} \mid 0\right)=Y_{\ell \ell^{\prime}}\left(\tau, \tau^{\prime} \mid \theta\right)>Y_{\ell \ell^{\prime}}\left(\tau, \tau^{\prime} \mid 0\right)=X_{\ell \ell^{\prime}}\left(\tau, \tau^{\prime} \mid 0\right) .
$$

This implies

$$
Y_{\ell \ell^{\prime}}^{(\theta)}\left(\tau, \tau^{\prime} \mid t\right)>0
$$

for all $\ell, \ell^{\prime} \in \Lambda, \tau, \tau^{\prime} \in[0, \beta]$ and $t \in[0,1-\theta]$.

Lemma 15 Let the condition (3.26) be satisfied. Then for all $\theta \in(0,1)$ and $\varepsilon \in$ $\left[0, \varepsilon_{0}\right)$, the solutions of the problems (3.23), (3.24) and (3.25) obey

$$
X_{\ell \ell^{\prime}}\left(\tau, \tau^{\prime} \mid t\right)<Y_{\ell \ell^{\prime}}^{(\theta)}\left(\tau, \tau^{\prime} \mid t\right),
$$

for all $\ell, \ell^{\prime} \in \Lambda, \tau, \tau^{\prime} \in[0, \beta]$ and $t \in[0,1-\theta]$.

Proof: Set

$$
Z_{\ell \ell^{\prime}}^{ \pm}\left(\tau, \tau^{\prime} \mid t\right)=Y_{\ell \ell^{\prime}}^{(\theta)}\left(\tau, \tau^{\prime} \mid t\right) \pm X_{\ell \ell^{\prime}}\left(\tau, \tau^{\prime} \mid t\right), \quad t \in[0,1-\theta]
$$

Then one has

$$
\begin{aligned}
& \frac{\partial}{\partial t} Z_{\ell \ell^{\prime}}^{-}\left(\tau, \tau^{\prime} \mid t\right)= \\
& =\frac{1}{2} \sum_{\ell_{1}, \ell_{2} \in \Lambda} J_{\ell_{1} \ell_{2}}^{\Lambda} \int_{0}^{\beta}\left[Z_{\ell \ell_{1}}^{+}\left(\tau, \tau_{1} \mid t\right) Z_{\ell^{\prime} \ell_{2}}^{-}\left(\tau^{\prime}, \tau_{1} \mid t\right)+Z_{\ell \ell_{1}}^{-}\left(\tau, \tau_{1} \mid t\right) Z_{\ell^{\prime} \ell_{2}}^{+}\left(\tau^{\prime}, \tau_{1} \mid t\right)\right] \mathrm{d} \tau_{1} \\
& \quad+\frac{\varepsilon}{|\Lambda|} \sum_{\ell_{1}, \ell_{2} \in \Lambda} \int_{0}^{\beta} Y_{\ell \ell_{1}}^{\theta}\left(\tau, \tau_{1} \mid t\right) Y_{\ell^{\prime} \ell_{2}}^{\theta}\left(\tau^{\prime}, \tau_{1} \mid t\right) \mathrm{d} \tau_{1}-T_{\ell \ell^{\prime}}\left(\tau, \tau^{\prime} \mid t\right)
\end{aligned}
$$


where $T_{\ell \ell^{\prime}}\left(\tau, \tau^{\prime} \mid t\right) \leqslant 0$ stands for the first term on the right-hand side of (3.23). By (3.34) and (3.31), we have

$$
Z_{\ell \ell^{\prime}}^{-}\left(\tau, \tau^{\prime} \mid 0\right)=Y_{\ell \ell^{\prime}}\left(\tau, \tau^{\prime} \mid \theta\right)-X_{\ell \ell^{\prime}}\left(\tau, \tau^{\prime} \mid 0\right)>0
$$

which has to hold for all $\ell, \ell^{\prime} \in \Lambda, \tau, \tau^{\prime} \in[0, \beta]$. For every $\ell, \ell^{\prime} \in \Lambda$, both $Y_{\ell \ell^{\prime}}\left(\tau, \tau^{\prime} \mid t\right)$, $X_{\ell \ell^{\prime}}\left(\tau, \tau^{\prime} \mid t\right)$, and hence $Z_{\ell \ell^{\prime}}^{ \pm}\left(\tau, \tau^{\prime} \mid t\right)$ are continuous functions of their arguments. For $Y_{\ell \ell^{\prime}}$, this follows from (3.27). For $X_{\ell \ell^{\prime}}$, this follows from its above-mentioned analyticity as a function of $t$ and its analyticity as a function of $\tau, \tau^{\prime}$, which was proven in Lemma 2.1 in [22]. Set

$$
\zeta(t)=\inf \left\{Z_{\ell \ell^{\prime}}^{-}\left(\tau, \tau^{\prime} \mid t\right) \mid \ell, \ell^{\prime} \in \Lambda, \quad \tau, \tau^{\prime} \in[0, \beta]\right\} .
$$

By (3.36), one has $\zeta(0)>0$. Suppose now that $\zeta\left(t_{0}\right)=0$ at some $t_{0} \in[0,1-\theta]$ and $\zeta(t)>0$ for $t \in\left[0, t_{0}\right)$. Then, by the continuity of $Z_{\ell \ell^{\prime}}^{-}$, there exists $\ell, \ell^{\prime} \in \Lambda$, $\tau, \tau^{\prime} \in[0, \beta]$ such that

$$
Z_{\ell \ell^{\prime}}^{-}\left(\tau, \tau^{\prime} \mid t_{0}\right)=0, \quad Z_{\ell \ell^{\prime}}^{-}\left(\tau, \tau^{\prime} \mid t\right)>0, \quad \text { for } t<t_{0}
$$

For these $\ell, \ell^{\prime} \in \Lambda, \tau, \tau^{\prime} \in[0, \beta]$, the derivative $(\partial / \partial t) Z_{\ell \ell^{\prime}}^{-}\left(\tau, \tau^{\prime} \mid t\right)$ at $t=t_{0}$ is positive since on the right-hand side of (3.35) the third term is positive by (3.32) and the rest of them are nonnegative. But a differentiable function, which is positive at $t \in\left[0, t_{0}\right)$ and zero at $t=t_{0}$, cannot increase at $t=t_{0}$. Thus, $\zeta(t)>0$ for all $t \in[0,1-\theta]$, which yields (3.33).

Corollary 16 Let $\Lambda$ be of the form (2.6) and let the condition (3.26) be satisfied. Then for all $\ell, \ell^{\prime} \in \Lambda, \tau, \tau^{\prime} \in[0, \beta]$, one has

$$
K_{\ell \ell^{\prime}}\left(\tau, \tau^{\prime} \mid \Lambda, p\right) \leqslant \frac{1}{\beta|\Lambda|} \sum_{\lambda \in \Lambda_{*}} \sum_{k \in \mathcal{K}} \frac{\exp \left[\mathrm{i} k\left(\tau-\tau^{\prime}\right)+\mathrm{i}\left(\lambda, \ell-\ell^{\prime}\right)\right]}{[U(k)]^{-1}-\hat{J}^{\Lambda}(0)+I^{\Lambda}(\lambda)} .
$$

Proof: For $t=1-\theta$, one has from (3.33), (3.30), (3.27)

$$
\begin{aligned}
& X_{\ell \ell^{\prime}}\left(\tau, \tau^{\prime} \mid 1-\theta\right)<Y_{\ell \ell^{\prime}}\left(\tau, \tau^{\prime} \mid 1\right)= \\
& \quad=\frac{1}{\beta|\Lambda|} \sum_{\lambda \in \Lambda_{*}} \sum_{k \in \mathcal{K}} \frac{\exp \left[\mathrm{i} k\left(\tau-\tau^{\prime}\right)+\mathrm{i}\left(\lambda, \ell-\ell^{\prime}\right)\right]}{[U(k)]^{-1}-\left(\hat{J}^{\Lambda}(0)+\varepsilon \delta_{\lambda, 0}\right)+I^{\Lambda}(\lambda)} .
\end{aligned}
$$

All the functions above depend on $\theta$ and $\varepsilon$ continuously. Hence, one may pass to the limit $\theta=\varepsilon \rightarrow 0$ and get (3.37).

Given $\ell, \ell^{\prime} \in \mathbb{L}$, one can find $L_{0} \in \mathbb{N}$ such that $\ell, \ell^{\prime}$ belong to the box $\Lambda_{L_{0}}(2.6)$.

Corollary 17 Let the condition (3.26) be satisfied. Then for the above $\ell, \ell^{\prime}$ and for all $\tau, \tau^{\prime} \in[0, \beta]$, the sequence $\left\{K_{\ell \ell^{\prime}}\left(\tau, \tau^{\prime} \mid \Lambda_{L}, p\right)\right\}_{L \geqslant L_{0}}$ is bounded. 
Proof: As it will be shown below, every correlation function (3.1) is nonnegative for all $\ell, \ell^{\prime} \in \Lambda$ and $\tau, \tau^{\prime} \in[0, \beta]$. On the other hand, the right-hand side of (3.37), converges as $L \rightarrow+\infty$ (i.e., as $\Lambda \rightarrow \mathbb{L}$ ) to the following limit

$$
\begin{aligned}
\lim _{L \rightarrow+\infty} & \operatorname{RHS}(3.37)= \\
\quad= & \frac{1}{\beta(2 \pi)^{d}} \sum_{k \in \mathcal{K}} \int_{(-\pi, \pi]^{d}} \frac{\exp \left[\mathrm{i} k\left(\tau-\tau^{\prime}\right)+\mathrm{i}\left(\lambda, \ell-\ell^{\prime}\right)\right]}{[U(k)]^{-1}-\hat{J}(0)+I(\lambda)} \mathrm{d} \lambda,
\end{aligned}
$$

hence, it is bounded.

Given a box $\Lambda$, let the matrix $M=\left(M_{\ell \ell^{\prime}}\right)_{\ell, \ell^{\prime} \in \Lambda}$ be such that $M_{\ell \ell^{\prime}}=M_{\ell^{\prime} \ell} \geqslant 0$. For two such matrices, we write $M^{\prime} \geqslant M$ meaning that $M_{\ell \ell^{\prime}}^{\prime} \geqslant M_{\ell \ell^{\prime}}$ for all $\ell, \ell^{\prime} \in \Lambda$. Set

$$
\begin{aligned}
\mathrm{d} \mu_{\Lambda}^{M}\left(x_{\Lambda}\right)= & \frac{1}{Z_{\beta, \Lambda}^{M}} \exp \left\{\frac{1}{2} \sum_{\ell, \ell^{\prime} \in \Lambda} M_{\ell \ell^{\prime}} \int_{0}^{\beta} x_{\ell}(\tau) x_{\ell^{\prime}}(\tau) \mathrm{d} \tau\right. \\
& \left.-\sum_{\ell \in \Lambda} \int_{0}^{\beta} V\left(x_{\ell}(\tau)\right) \mathrm{d} \tau\right\} \mathrm{d} \chi_{\Lambda}\left(x_{\Lambda}\right),
\end{aligned}
$$

where $1 / Z_{\beta, \Lambda}^{M}$ is the normalizing factor. Set also

$$
K_{\ell \ell^{\prime}}^{M}\left(\tau, \tau^{\prime} \mid \Lambda\right)=\int_{\mathcal{C}_{\Lambda}} x_{\ell}(\tau) x_{\ell^{\prime}}\left(\tau^{\prime}\right) \mathrm{d} \mu_{\Lambda}^{M}\left(x_{\Lambda}\right) .
$$

The next statement is a direct corollary of the second GKS inequality, which for measures like (3.39), was proven in [22], Theorem 6.2.

Proposition 18 If $M \leqslant M^{\prime}$, then

$$
K_{\ell \ell^{\prime}}^{M}\left(\tau, \tau^{\prime} \mid \Lambda\right) \leqslant K_{\ell \ell^{\prime}}^{M^{\prime}}\left(\tau, \tau^{\prime} \mid \Lambda\right),
$$

which holds for all $\ell, \ell^{\prime} \in \Lambda$ and $\tau, \tau^{\prime} \in[0, \beta]$.

Corollary 19 For every box $\Lambda$, for any $\ell, \ell^{\prime} \in \Lambda$ and $\tau, \tau^{\prime} \in[0, \beta]$,

$$
K_{\ell \ell^{\prime}}\left(\tau, \tau^{\prime} \mid \Lambda, 0\right) \leqslant K_{\ell \ell^{\prime}}\left(\tau, \tau^{\prime} \mid \Lambda, p\right)
$$

For any two boxes $\Lambda, \Lambda^{\prime}$, such that $\Lambda \subset \Lambda^{\prime}$,

$$
K_{\ell \ell^{\prime}}\left(\tau, \tau^{\prime} \mid \Lambda, 0\right) \leqslant K_{\ell \ell^{\prime}}\left(\tau, \tau^{\prime} \mid \Lambda^{\prime}, 0\right),
$$

which holds for all $\ell, \ell^{\prime} \in \Lambda$ and $\tau, \tau^{\prime} \in[0, \beta]$.

Proof: By (2.8), $\left|\ell-\ell^{\prime}\right|_{\Lambda_{L}} \leqslant\left|\ell-\ell^{\prime}\right|$, which by (2.7), (2.9) yields $J_{\ell \ell^{\prime}} \leqslant J_{\ell \ell^{\prime}}^{\Lambda}$. Hence, (3.42) follows from (3.41). For $\Lambda \subset \Lambda^{\prime}$ and $\ell, \ell^{\prime} \in \Lambda^{\prime}$, we set

$$
\tilde{J}_{\ell \ell^{\prime}}= \begin{cases}J_{\ell \ell^{\prime}}, & \text { if } \quad \ell, \ell^{\prime} \in \Lambda \\ 0, & \text { otherwise }\end{cases}
$$


Then $\tilde{J}_{\ell \ell^{\prime}} \leqslant J_{\ell \ell^{\prime}}$ for all $\ell, \ell^{\prime} \in \Lambda^{\prime}$. On the other hand,

$$
K_{\ell \ell^{\prime}}\left(\tau, \tau^{\prime} \mid \Lambda, 0\right)=K_{\ell \ell^{\prime}}^{\tilde{J}}\left(\tau, \tau^{\prime} \mid \Lambda^{\prime}, 0\right)
$$

then (3.43) also follows from (3.41).

Proof of Theorem 9: The nonnegativity of the correlation functions (3.1) follows form the first GKS inequality, proven in [22], Theorem 6.2. By (3.17), the condition (3.26) will be satisfied if (3.7) holds. Given $\ell, \ell^{\prime} \in \mathbb{L}$, we pick up $L_{0} \in \mathbb{N}$ such that $\ell, \ell^{\prime} \in \Lambda=\Lambda_{L_{0}}$. Then by (3.42), (3.43)

$$
K_{\ell \ell^{\prime}}\left(\tau, \tau^{\prime} \mid \Lambda, 0\right) \leqslant K_{\ell \ell^{\prime}}\left(\tau, \tau^{\prime} \mid \Lambda^{\prime}, p\right)
$$

which holds for any $\Lambda^{\prime}=\Lambda_{L}, L \geqslant L_{0}$, for all $\ell, \ell^{\prime} \in \Lambda$ and $\tau, \tau^{\prime} \in[0, \beta]$. Passing here to the limit $L \rightarrow+\infty$ and taking into account (3.37), (3.38) one arrives at (3.8) with $\Xi_{\beta}(k)=[U(k)]^{-1}-\hat{J}(0)$.

Proof of Theorem 10: By (3.8), the sequence $\left\{K_{\ell \ell^{\prime}}\left(\tau, \tau^{\prime} \mid \Lambda_{L}, 0\right)\right\}_{L \geqslant L_{0}}$ is bounded and by (3.43), it is monotone increasing, hence convergent. Its limit does not exceed the bound (3.8).

Proof of Corollary 12: Given $\Lambda$, one has by (3.37)

$$
\begin{aligned}
\frac{1}{|\Lambda|} \sum_{\ell, \ell^{\prime} \in \Lambda} \int_{0}^{\beta} K_{\ell \ell^{\prime}}(0, \tau \mid \Lambda, p) \mathrm{d} \tau & \leqslant \frac{1}{[U(0)]^{-1}-\hat{J}^{\Lambda}(0)} \\
& \leqslant \frac{1}{[U(0)]^{-1}-\hat{J}(0)}<\infty
\end{aligned}
$$

which holds in view of (3.26). By (3.11), (3.42), (3.43), one has

$$
\varpi_{\Lambda} \leqslant \frac{1}{|\Lambda|} \sum_{\ell, \ell^{\prime} \in \Lambda} \int_{0}^{\beta} K_{\ell \ell^{\prime}}(0, \tau \mid \Lambda, p) \mathrm{d} \tau \leqslant \frac{1}{[U(0)]^{-1}-\hat{J}(0)}
$$

and $\varpi_{\Lambda} \leqslant \varpi_{\Lambda^{\prime}}$ for $\Lambda \subset \Lambda^{\prime}$. Therefore, the sequence $\left\{\varpi_{\Lambda_{L}}\right\}_{L \in \mathbb{N}}$ is monotone increasing and bounded, hence convergent to a certain $\varpi_{\infty} \leqslant\left[[U(0)]^{-1}-\hat{J}(0)\right]^{-1}$.

As it has been mentioned earlier, the spatial decay of the right-hand side of (3.8) is exponential if $I(\lambda) \sim j|\lambda|^{2}$ as $\lambda \rightarrow 0$. Let us prove this directly in the case of $J_{\ell \ell^{\prime}}=J \delta_{\left|\ell-\ell^{\prime}\right|, 1}, J>0$. Namely, we are going to show that

$$
\operatorname{RHS}(3.8) \leqslant C_{\beta} \exp \left(-\alpha_{0}\left|\ell-\ell^{\prime}\right|\right)
$$

with

$$
\begin{aligned}
\alpha_{0} & =d^{-1 / 2} \ln \left(1+\frac{m \Delta^{2}-2 d J}{2 J}\right) \\
C_{\beta} & =\frac{1}{\beta} \sum_{k \in \mathcal{K}} \frac{1}{m \Delta^{2}-2 d J-m k^{2}} .
\end{aligned}
$$


To this end we use a known formula

$$
\begin{aligned}
\frac{1}{2 \pi} \int_{-\pi}^{\pi} \frac{\mathrm{e}^{\mathrm{i} t l} \mathrm{~d} t}{a-\cos t} & =\frac{1}{\sqrt{a^{2}-1}} \exp (-\alpha|l|), \\
\alpha & =\ln \left(a+\sqrt{a^{2}-1}\right),
\end{aligned}
$$

which holds for all $a>1$ and $l \in \mathbb{Z}$. Now we choose the component of $\ell-\ell^{\prime}$ with the biggest absolute value. Suppose it is $\ell_{j}-\ell_{j}^{\prime}$. Set $\hat{\ell}, \hat{\ell}^{\prime}$ and $\hat{\lambda}$ to be the $d-1-$ dimensional vectors which one obtains from the corresponding $d$-dimensional vectors by excluding the $j$-ths components. Then by (3.47)

$$
\begin{aligned}
\operatorname{RHS}(3.8)= & \frac{1}{2 J \beta(2 \pi)^{d-1}} \sum_{k \in \mathcal{K}} \int_{(-\pi, \pi]^{d-1}} \exp \left[\mathrm{i} k\left(\tau-\tau^{\prime}\right)+\mathrm{i}\left(\hat{\lambda}, \hat{\ell}-\hat{\ell^{\prime}}\right)\right] \mathrm{d} \hat{\lambda} \\
& \times \frac{1}{\sqrt{a^{2}-1}} \exp \left(-\alpha\left|\ell_{j}-\ell_{j}^{\prime}\right|\right)
\end{aligned}
$$

where $\alpha$ is as in (3.47) with

$$
a=\frac{1}{2 J}[U(k)]^{-1}-\sum_{i=1, i \neq j}^{d} \cos \lambda_{i}>1,
$$

which holds in view of (3.26). Applying here the estimate (3.17) and the following evident facts

$$
\left|\ell_{j}-\ell_{j}^{\prime}\right| \geqslant d^{-1 / 2}\left|\ell-\ell^{\prime}\right|, \quad \sqrt{a^{2}-1}>a-1, \quad \text { for } a>1,
$$

one arrives at (3.45), (3.46).

\section{Acknowledgements}

Yuri Kozitsky was financially supported by the Komitet Badań Naukowych through the Grant 2 P03A 020 25, which is cordially acknowledged.

\section{References}

1. Vaks V.G. Introduction to the Macroscopic Theory of Ferroelectrics. Moscow, Nauka, 1973 (in Russian).

2. Driessler W., Landau L., Perez J.F. // J. Stat. Phys., 1979, vol. 20, p. 123-162.

3. Pastur L.A., Khoruzhenko B.A. // Teor. Math. Fiz., 1987, vol. 73. p. 111-124.

4. Stamenković S. // Condens. Matter Phys., 1998, vol. 1, No. 2(14), p. 257-309.

5. Freericks J.K., Jarrel M., Mahan G.D. // Phys. Rev. Lett., 1996, vol. 77, p. 4588-4591.

6. Dyson F.J., Lieb E.H., Simon B. // J. Stat. Phys., 1978, vol. 18, p. 335-383.

7. Simon B. The Statistical Mechanics of Lattice Gases. Princeton, New Jersey, Princeton University Press, 1993.

8. Albeverio S., Høegh-Krohn R. Theory of Feynman Path Integrals. Lecture Notes in Math. 523, Berlin, New York, Springer, 1976. 
9. Simon B. Functional Integration and Quantum Physics. New York, San Francisco, London, Academic Press, 1979.

10. Roepstorff G. Path Integral Approach to Quantum Physics. Berlin, Heidelberg, Springer-Verlag, 1993.

11. Høegh-Krohn R. // Commun. Math. Phys., 1974, vol. 38, p. 195-224.

12. Albeverion S., Høegh-Krohn R. // J. Func. Anal., 1975, vol. 19, p. 242-279.

13. Barbulyak V.S., Kondratiev Yu.G. // Rep. Nat. Acad. Sci of Ukraine, 1991, No. 9, p. $38-40$.

14. Barbulyak V.S., Kondratiev Yu.G. // Rep. Nat. Acad. Sci of Ukraine, 1991, No. 8, p. 31-34.

15. Barbulyak V.S., Kondratiev Yu.G. // Rep. Nat. Acad. Sci of Ukraine, 1991, No. 10, p. 19-21.

16. Barbulyak V.S., Kondratiev Yu.G. // Func. Anal. Appl., 1992, vol. 26(2), p. 61-64.

17. Globa S.A., Kondratiev Yu. G. // Selecta Math. Sov., 1990, vol. 9, p. 297-307.

18. Kozitsky Yu. Gibbs states of a lattice system of quantum anharmonic oscillators. - In: Noncommutative Structures in Mathematics and Physics, Kiev, 2000, p. 415-425, (S. Duplij and J. Wess eds) NATO Sci Ser. II Math. Phys. Chem. 22. Dordrecht, Kluwer Acad. Publ., 2001.

19. Albeverio S., Kondratiev Yu., Kozitsky Yu. // Commun. Math. Phys., 1998, vol. 194, p. 493-512.

20. Albeverio S., Kondratiev Yu., Kozitsky Yu. // Lett. Math. Phys., 1999, vol. 48, p. 221233.

21. Albeverio S., Kondratiev Yu., Kozitsky Yu., Röckner M. // Ann. Inst. H. Poincaré, 2001, vol. 37, p. 43-69.

22. Albeverio S., Kondratiev Yu., Kozitsky Yu., Röckner M. // Rev. Math. Phys., 2002, vol. 14, p. 1335-1401.

23. Albeverio S., Kondratiev Yu., Kozitsky Yu., Röckner M. // C. R. Acad. Sci. Paris, Ser. I, 2002, vol. 335, p. 693-698.

24. Albeverio S., Kondratiev Yu., Kozitsky Yu., Röckner M. // Phys. Rev. Lett., 2003, vol. 90, No. 17, p. 170603-1-4.

25. Albeverio S., Kondratiev Yu., Röckner M., Tsikalenko T. // Probab. Theory Relat. Fields, 1997, vol. 108, p. 193-218.

26. Albeverio S., Kondratiev Yu., Röckner M., Tsikalenko T. // Commun. Math. Phys., 1997, vol. 189, p. 621-630.

27. Albeverio S., Kondratiev Yu., Röckner M., Tsikalenko T. // Rev. Math. Phys., 2001, vol. 13 , p. $51-124$.

28. Kozitsky Yu. // Condens. Matter Phys., 2002, vol. 5, No. 4(32), p. 601-616.

29. Bratteli O., Robinson D. W. Operator Algebras and Quantum Statistical Mechanics, I, II. New York, Springer-Verlag, 1981.

30. Berezin F.A., Shubin M.A. The Schrödinger Equation. Dordrecht, Boston, London, Kluwer Academic Publishers, 1991.

31. Reed M., Simon B. Methods of Modern Mathematical Physics. I. Functional Analysis. New York London, Academic Press, 1972.

32. Birke L., Fröhlich J. // Rev. Math. Phys., 2002, vol. 14, p. 829-871.

33. Georgii H.-O. Gibbs Measures and Phase Transitions. Vol. 9. Berlin, New York, Walter de Gruyter, 1988.

34. Albeverio S., Kondratiev Yu., Pasurek T., Röckner M. // Ann. Probab. (in print). 
35. Blinc R., Žekš B. Soft Modes in Ferroelectrics and Antiferroelectrics, Amsterdam, North-Holland, 1974.

36. Tibbals J.E., Nelmes R.J., McIntyre G.J. // J. Phys. C: Solid State Phys., 1982, vol. 15 , p. $37-58$.

37. Schneider T., Beck H., Stoll E. // Phys. Rev. B, 1976, vol. 13, p. 1123-1130.

38. Plakida N.M., Tonchev M.S. // Phys. A, 1986, vol. 136, p. 176-188.

39. Verbeure A., Zagrebnov V.A. // J. Statist. Phys., 1992, vol. 69, p. 37-55.

40. Verbeure A., Zagrebnov V.A. // J. Phys. A: Math. Gen., 1995, vol. 28, p. 5415-5421.

41. Minlos, R.A., Verbeure A., Zagrebnov V.A. // Rev. Math. Phys., 2000, vol. 12, p. 981.

42. Glimm, J., Jaffe, A. Quantum Physics: A Functional Integral Point of View. 2nd edn. New York, Springer-Verlag, 1987.

\title{
Квантовий ангармонічний кристал у підході, що базується на функціональних інтегралах
}

\author{
Ю.Кондратьєв ${ }^{1,3,4}$, Ю.Козицький ${ }^{2}$, Т.Пазурек ${ }^{1,3}$, \\ М.Рекнер ${ }^{1,3}$ \\ 1 Математичний факультет університету Білефельд, \\ Німеччина, D 33615 Білефельд \\ 2 Інститут математики, \\ Університет Марії Кюрі-Склодовської в Любліні, \\ Польща, PL 20-031 Люблін \\ 3 Дослідницький центр BiBoS, Німеччина, Білефельд \\ 4 Інститут математики НАН України, Київ
}

Отримано 15 жовтня 2003 р.

В рамках підходу, що базується на функціональних інтегралах, розглядається ґраткова модель взаємодіючих квантових частинок маси $m$, які осцилюють у кристалічному полі. Головні аспекти такого підходу описуються у спосіб доступний для неспеціалістів. У результаті запропоновано механізм стабілізації моделі, спричиненої квантовими ефектами. Зокрема, дається умова стабілізації, що включає в себе $m$, інтенсивність взаємодії та параметри кристалічного поля. Ця умова не залежить від температури, вона задовільняється, якщо $m \in$ достатньо малою та/або частота тунелювання $є$ достатньо великою. Показано, що за цієї умови кореляційні функції, обчислені у термодинамічній границі, спадають експоненційно, що унеможливлює фазові переходи при всіх температурах.

Ключові слова: квантова стабілізація, стани Гіббса, м'яка мода

PACS: $05.50 .-d, 64.60 .-i$ 